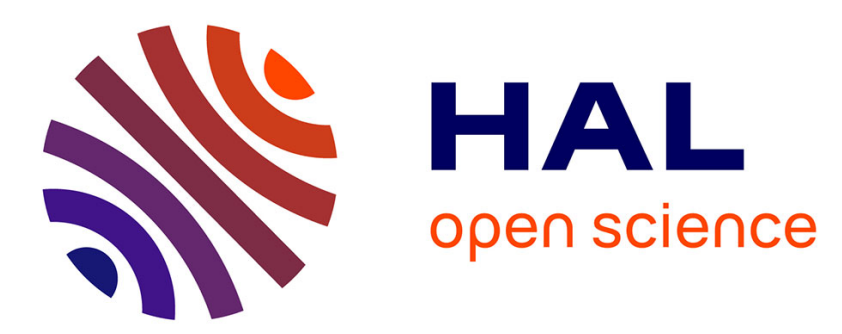

\title{
Model reduction and perturbation analysis of wave finite element formulations for computing the forced response of coupled elastic systems involving junctions with uncertain eigenfrequencies \\ Jean-Mathieu Mencik
}

\section{To cite this version:}

Jean-Mathieu Mencik. Model reduction and perturbation analysis of wave finite element formulations for computing the forced response of coupled elastic systems involving junctions with uncertain eigenfrequencies. Computer Methods in Applied Mechanics and Engineering, 2011, 200, pp.3051-3065. 10.1016/j.cma.2011.06.014 . hal-00755754

HAL Id: hal-00755754

https://hal.science/hal-00755754

Submitted on 21 Nov 2012

HAL is a multi-disciplinary open access archive for the deposit and dissemination of scientific research documents, whether they are published or not. The documents may come from teaching and research institutions in France or abroad, or from public or private research centers.
L'archive ouverte pluridisciplinaire HAL, est destinée au dépôt et à la diffusion de documents scientifiques de niveau recherche, publiés ou non, émanant des établissements d'enseignement et de recherche français ou étrangers, des laboratoires publics ou privés. 


\title{
Model reduction and perturbation analysis of wave finite element formulations for computing the forced response of coupled elastic systems involving junctions with uncertain eigenfrequencies
}

\author{
J.-M. Mencik \\ ENI Val de Loire, Université François Rabelais de Tours, LMR laboratory, Rue de la Chocolaterie, \\ BP 3410, F-41034 Blois Cedex, France
}

\begin{abstract}
The wave finite element method is investigated for computing the low- and midfrequency forced response of coupled elastic systems involving straight structures with junctions. The relevance of the method is discussed when a component mode synthesis procedure is used for modeling the junctions. A norm-wise selection criterion is proposed so as to reduce efficiently the number of junction modes retained in the wave-based formulations. Component-wise perturbation bounds of the wave-based displacement / force solutions are also derived to address slight uncertainties for the junction eigenfrequencies. Numerical comparisons with standard finite element solutions as well as Monte Carlo simulations highlight the relevance of the formulation.
\end{abstract}

Key words: Wave finite elements, component mode synthesis, model reduction, perturbation analysis.

Email address: jean-mathieu.mencik@univ-tours.fr(J.-M. Mencik) 


\section{Introduction}

This paper aims at investigating the wave finite element (WFE) method for computing the low- and mid-frequency (LF and MF) forced response of coupled systems involving straight beam-like structures - namely waveguides - with elastic junctions. Such systems are extensively encountered in many engineering areas such as those involved in the manufacturing of chassis frames in automotive industry, among others. For example, the case of two waveguides connected with a quarter of torus (junction) is depicted in Figure (1). Within the MF range, the waveguide cross-sections, as well as the junctions, are expected to undergo oscillating spatial dynamics and resonances. It turns out that the behavior of these coupled systems is expected to involve both global waveguide resonances (i.e. induced by the system left and right boundaries) and local cross-section / junction resonances whose related modal densities can exhibit large variations [1, 2].

The strategy for computing the LF and MF forced response of elastic systems by means of WFE matrix formulations has been recently proposed in ref. [3] (other approaches can be found in refs. [4, 5]). In this framework, reduced bases of numerical wave modes with one-dimensional propagation (but not necessarily uniform cross-section shapes ${ }^{1}$ ) are used to span the kinematic fields of waveguides. The key feature of these wave-based matrix formulations is that the use of "natural" wave motions as representation bases enforces their convergence, even if reduced bases of relatively small dimensions are dealt with. In other words, these matrix formulations exhibit sizes that are expected to be much smaller compared to what is encountered when using other types of reduced-order models (e.g. component mode synthesis [7], Krylov subspace techniques [8] or proper orthogonal decom-

\footnotetext{
${ }^{1}$ These wave shapes are provided using a discretization scheme for the cross-section that results from a finite element (FE) modeling of a small substructure [6].
} 
position [9]), yielding extra CPU time savings. The strategy for addressing the forced response of coupled systems has also been proposed in ref. [3, 10]. In this framework, a FE mesh tying procedure between waveguides and junctions, based on Lagrange Multipliers, is used for computing wave reflection / transmission coefficients. The relevance of the technique has been highlighted in ref. [3] for addressing the system depicted in Figure (1); the drawback of this analysis is that the response of the junction has only been investigated as static.

The present study aims at investigating the WFE method further on in the frequency domain for computing the forced response of coupled systems involving resonant junctions. The two following topics are particularly addressed: (i) a component mode synthesis (CMS) procedure so as to describe the frequency behavior of junctions with a small number of elastic modes; (ii) a perturbation analysis so as to address slight uncertainties for the junction eigenfrequencies. The concepts of CMS techniques and perturbation analysis are not new and have been widely treated in the literature in different kinds of FE problems. However, their application to wave-based matrix formulations does not seem straightforward. In fact, a WFE-based CMS / Craig Bampton (CB) procedure that uses junction modes for computing wave reflection / transmission coefficients has already been proposed in ref. [11]. A quite similar approach has also been proposed in ref. [12] in the framework of the spectral element method (SEM) for computing time responses of systems involving two-node waveguides with FE junctions. However, regarding these works, a question arises as to how selecting an optimal reduced family of junction modes for computing the system forced response with accurate precision. This task addresses model order reduction procedures with a view to saving additional CPU time when solving the WFE formulations ${ }^{2}$. Several works have been carried

\footnotetext{
${ }^{2}$ This appears especially attractive in the framework of parametric studies, the junction modes
} 
out on that issue concerning classical substructuring approaches, where norm-wise estimators are invoked as selection criteria of component modes [13, 14, 15]. The feature of these approaches is that the mode selection can be performed in a single pre-processing step, without the need for computing the forced responses. In other words, these approaches appear advantageous compared to the usual empiric strategy that consists in selecting the modes in "ascending order", i.e. using arbitrary numbers of modes as test cases in several time consuming post-processing steps. The way such norm-wise criteria can be applied within the WFE framework constitutes an interesting challenge which had never been investigated so far. Otherwise, perturbation analysis of the system forced response seems to constitute another open challenge within the WFE framework that addresses uncertainty propagation and interval analyzes [16].

The issues mentioned above are addressed in the present study. For the sake of clarity, the case of two waveguides connected with a single junction is considered (cf. Figure 1). The paper is organized as follows. The WFE strategy for computing the forced response of the coupled system is briefly recalled in Section 2 according to previous works $[3,10,17]$; additional statements and proofs are brought with regard to the computation of the reflection and transmission coefficients. Section 3 addresses the CMS / CB procedure for describing the junction dynamics; a wavebased norm-wise criterion is proposed for selecting the junction modes that are of primary interest for computing the forced response of the coupled system; the relevance of this strategy is highlighted when compared to the conventional procedure that consists in sorting the modes in ascending order. Section 4 finally addresses perturbation analysis for computing WFE solutions when slight uncertainties affect the junction eigenfrequencies. Component-wise bounds are formulated in this

$\overline{\text { being considered as independent input data. }}$ 
sense. The relevance of the strategy is discussed through comparisons with Monte Carlo simulations (MCS).

\section{Figure 1}

\section{WFE method}

\subsection{Basic concepts}

The WFE method aims at providing numerically the LF and MF wave propagation into periodic elastic structures [18]. In the present study, these structures are supposed to be dissipative with a loss factor $\eta$ and subjected to harmonic disturbance under frequency $\omega / 2 \pi$ (where $\omega$ is the pulsation). It is assumed that each structure is described from a set of identical substructures. These are modeled by means of the same FE model and connected along a principal axis $x$ referred to as the direction of propagation (see Figure 2). The length of each substructure, along this direction, is denoted as $d$. Also, it is assumed that both left and right crosssections of each substructure are discretized in the same way, i.e. they contain the same number $n$ of degrees of freedom (DOFs).

\section{Figure 2}

The WFE method requires the mass and stiffness matrices $(\mathbf{M}, \mathbf{K})$ of a typical substructure (see Figure 2) to be known; it uses a state vector representation [6] for linking the kinematic variables - i.e. displacements $\mathbf{q}$ and forces $\mathbf{F}$ - expressed over the left or right cross-section of a substructure $k$ to those expressed over a similar cross-section (either left or right) for an adjacent substructure $k-1$. In the frequency domain, this relationship is expressed in terms of a $2 n \times 2 n$ symplectic matrix $\mathbf{S}$ as [3]:

$$
\mathbf{u}^{(k)}=\mathbf{S u}^{(k-1)},
$$


where $\mathbf{u}$ is a $2 n \times 1$ state vector expressed as:

$$
\mathbf{u}=\left[\begin{array}{c}
\mathbf{q} \\
\pm \mathbf{F}
\end{array}\right]
$$

According to the action-reaction law, the sign of $\mathbf{F}$ in Eq. (2) needs to be switched depending on which left or right cross-section is considered ${ }^{3}$. In Eq. (1), $\mathbf{S}$ formally reflects a translation operator between state vectors $\mathbf{u}^{(k-1)}$ and $\mathbf{u}^{(k)}$, say between abscissa $x_{k-1}$ and $x_{k}=x_{k-1}+d$. The wave mode computation follows directly from Bloch's theorem [19]:

Bloch's theorem: a simple statement. Let $\mathbf{S}$ be d-periodic, it turns out that $\mathbf{u}^{(k)}$ can be expanded as $\sum_{j} Q_{j}^{(k)} \boldsymbol{\Phi}_{j}$ where $Q_{j}^{(k)}=\mathrm{e}^{-\mathrm{i} \beta_{j} d} Q_{j}^{(k-1)} \forall j$.

The terms $\left\{Q_{j}^{(k)} \boldsymbol{\Phi}_{j}\right\}_{j}$ are usually called the eigenstates of S. According to Bloch's theorem, these represent waves traveling along the structure as $\left\{\mathrm{e}^{-i \beta_{j} d}\right\}_{j}$. Bloch's theorem particularly states that eigenvalues of $\mathbf{S}$ - namely $\left\{\mu_{j}\right\}_{j}-$ can be expressed as $\left\{\mathrm{e}^{-\mathrm{i} \beta_{j} d}\right\}_{j}$ where $\left\{\beta_{j}\right\}_{j}$ have the meaning of wavenumbers. Also, the terms $\left\{\boldsymbol{\Phi}_{j}\right\}_{j}$ are the eigenvectors of $\mathbf{S}$ - also called the wave shapes - that relate spatial distributions of displacements and internal forces over the structure cross-section. The wave modes of the waveguide are defined as $\left\{\left(\mu_{j}, \mathbf{\Phi}_{j}\right)\right\}_{j}$. According to the symplectic nature of $\mathbf{S}$ and since dissipative structures are considered, they can be readily classified as $\left\{\mu_{j}^{\text {inc }}\right\}_{j=1, \ldots, n}=\left\{\mu_{j}:\left|\mu_{j}\right|<1\right\}_{j}$ and $\left\{\mu_{j}^{\text {ref }}\right\}_{j=1, \ldots, n}=\left\{\mu_{j}:\left|\mu_{j}\right|>1\right\}_{j}$, where superscripts inc and ref respectively denote the incident and reflected waves (see Figure 2$)^{4}$. As a result, the wave

\footnotetext{
${ }^{3}$ The conventions $-\mathbf{F}_{L}$ and $\mathbf{F}_{R}$ are usually stated for the left and right cross-sections, respectively.

${ }^{4}$ The terms "incident" and "reflected" denote the waves traveling in the $x$-positive and $x-$ negative directions (respectively), i.e. the waves traveling towards and outwards the right waveg-
} 
shapes $\left\{\boldsymbol{\Phi}_{j}\right\}_{j}$ can be expressed in matrix form as:

$$
\boldsymbol{\Phi}=\left[\begin{array}{cc}
\boldsymbol{\Phi}_{\mathrm{q}}^{\text {inc }} & \boldsymbol{\Phi}_{\mathrm{q}}^{\mathrm{ref}} \\
\boldsymbol{\Phi}_{\mathrm{F}}^{\text {inc }} & \boldsymbol{\Phi}_{\mathrm{F}}^{\mathrm{ref}}
\end{array}\right],
$$

i.e. in terms of square $n \times n$ matrices $\Phi_{\mathrm{q}}^{\text {inc }}, \boldsymbol{\Phi}_{\mathrm{F}}^{\text {inc }}, \Phi_{\mathrm{q}}^{\text {ref }}$ and $\boldsymbol{\Phi}_{\mathrm{F}}^{\text {ref }}$, where the subscripts q / F refer to as displacement / force components.

For example, the displacement components for several wave shapes are plotted in Figure 3 when considering the beam-like structure case depicted in Figure 2. These shapes represent the usual LF wave motions (i.e. flexural, torsional, longitudinal and shearing) and additional modes. The latter are not necessarily assessed by means of analytical theories but play a crucial role for describing the local structure dynamics in the MF domain. Apart from the prediction of these MF modes, the feature of the WFE method is that it is not frequency-limited by LF analytical assumptions (e.g. plane cross-sections). This is explained as it uses a FE discretization scheme for addressing the cross-section dynamics in the short wavelength domain.

\section{Figure 3}

Bloch's theorem also states that each state vector $\mathbf{u}^{(k)}$ can be expanded as $\sum_{j} Q_{j}^{(k)} \boldsymbol{\Phi}_{j}$ where $\left\{\boldsymbol{\Phi}_{j}\right\}_{j}$ and $\left\{Q_{j}^{(k)}\right\}_{j}$ play the role of wave basis (with dimension $2 n$ ) and modal amplitudes, respectively. As a rule of thumb, such an expansion is carried out with a reduced basis of wave shapes $\left\{\widetilde{\boldsymbol{\Phi}}_{j}\right\}_{j=1, \ldots, 2 m}$ only, extracted from the full basis $\left\{\boldsymbol{\Phi}_{j}\right\}_{j=1, \ldots, 2 n}$ and with the same number $m(m \leq n)$ of incident and reflected modes. The strategy for selecting such a reduced basis is discussed in ref.

uide boundary. 
[3]. In matrix form, it turns out that wave expansion writes as:

$\mathbf{q}^{(k)} \approx \widetilde{\boldsymbol{\Phi}}_{\mathbf{q}}^{\text {inc }} \widetilde{\mathbf{Q}}^{\text {inc }(k)}+\widetilde{\boldsymbol{\Phi}}_{\mathrm{q}}^{\text {ref }} \widetilde{\mathbf{Q}}^{\text {ref }(k)} \quad, \quad \pm \mathbf{F}^{(k)} \approx \widetilde{\boldsymbol{\Phi}}_{\mathrm{F}}^{\text {inc }} \widetilde{\mathbf{Q}}^{\text {inc }(k)}+\widetilde{\boldsymbol{\Phi}}_{\mathrm{F}}^{\text {ref }} \widetilde{\mathbf{Q}}^{\text {ref }(k)} \quad \forall k$,

while the spatial distributions of the modal amplitudes $\widetilde{\mathbf{Q}}^{\text {inc }(k)}$ and $\widetilde{\mathbf{Q}}^{\mathrm{ref}(k)}$ are governed as:

$$
\widetilde{\mathbf{Q}}^{\text {inc }(k)}=\widetilde{\boldsymbol{\mu}}^{k-1} \widetilde{\mathbf{Q}}^{\operatorname{inc}(1)} \quad, \quad \widetilde{\mathbf{Q}}^{\mathrm{ref}(k)}=\widetilde{\boldsymbol{\mu}}^{-(k-1)} \widetilde{\mathbf{Q}}^{\mathrm{ref}(1)} \quad \forall k .
$$

Here, $\widetilde{\boldsymbol{\mu}}$ represents the $m \times m$ diagonal eigenvalue matrix of the incident modes, defined as $\widetilde{\boldsymbol{\mu}}=\widetilde{\boldsymbol{\mu}}^{\text {inc }}=\left(\widetilde{\boldsymbol{\mu}}^{\text {ref }}\right)^{-1}[3]$.

\subsection{Mesh tying problem and wave-based coupling conditions}

The problem of predicting the behavior of coupled waveguides using the WFE method is addressed. Such a problem is depicted in Figure 1 and represents two waveguides 1 and 2 connected with an elastic junction over two coupling interfaces $\Gamma_{1}$ and $\Gamma_{2}$, where mesh compatibility is not necessarily verified. The underlying mesh tying problem is illustrated in Figure 4 and concerns two substructures, for waveguides 1 and 2, connected with a junction whose internal DOFs are considered to be free from excitation sources. The fact that mesh compatibility is not assumed means that the number of DOFs used for discretizing the cross-section of each waveguide $i$ - say $n_{i}$ - is not necessarily equal to the number of DOFs used for discretizing the coupling junction over $\Gamma_{i}$.

\section{Figure 4}

The use of non-compatible meshes can be addressed by means of Lagrange Multipliers combined with the Mortar method [20], where the continuity of displacements across $\Gamma_{1}$ and $\Gamma_{2}$ is imposed in a weak sense. In this framework, the 
interpolation functions of the Lagrange Multiplier fields are chosen so that they correspond to the trace of the junction interpolation functions over $\Gamma_{1}$ and $\Gamma_{2}$. This yields a unique way to express the displacements of the junction from those of the connected substructures, and conversely the forces applied to the substructures from those applied to the junction $[10,17]$. Using superscript / subscript notations $\mathrm{c}$ and $i$ as references to the coupling junction and the waveguide $i$ (respectively) yields:

$$
\left.\mathbf{q}^{\mathrm{c}}\right|_{\Gamma_{i}}=\left.\left(\mathcal{B}_{\mathrm{c}}^{(i)}\right)^{-1} \mathcal{B}_{i}^{(i)} \mathbf{q}_{i}\right|_{\Gamma_{i}} \quad,\left.\quad \mathbf{F}_{i}\right|_{\Gamma_{i}}=-\left.\left(\mathcal{B}_{i}^{(i)}\right)^{T}\left(\mathcal{B}_{\mathrm{c}}^{(i)}\right)^{-T} \mathbf{F}^{\mathrm{c}}\right|_{\Gamma_{i}} \quad i=1,2,
$$

where $\left\{\mathcal{B}_{\mathrm{c}}^{(i)}\right\}_{i=1,2}$ are square positive definite matrices defined in refs. [10, 17], while $\left.\mathbf{q}_{i}\right|_{\Gamma_{i}}$ and $\left.\mathbf{q}^{\mathrm{c}}\right|_{\Gamma_{i}}$ (resp. $\left.\mathbf{F}_{i}\right|_{\Gamma_{i}}$ and $\left.\mathbf{F}^{\mathrm{c}}\right|_{\Gamma_{i}}$ ) denote the displacements (resp. the forces) of waveguide $i$ and junction over coupling interface $\Gamma_{i}$. In matrix form, Eq. (6) results in

$$
-\mathbf{T}^{T} \mathbb{D}^{*} \mathbf{T}\left[\begin{array}{c}
\left.\mathbf{q}_{1}\right|_{\Gamma_{1}} \\
\left.\mathbf{q}_{2}\right|_{\Gamma_{2}}
\end{array}\right]=\left[\begin{array}{c}
\left.\mathbf{F}_{1}\right|_{\Gamma_{1}} \\
\left.\mathbf{F}_{2}\right|_{\Gamma_{2}}
\end{array}\right],
$$

where $\mathbf{T}$ is a block diagonal real matrix whose components are $\left\{\left(\mathcal{B}_{\mathrm{c}}^{(i)}\right)^{-1} \mathcal{B}_{i}^{(i)}\right\}_{i=1,2}$ and which results from the use of Lagrange multipliers in the mesh tying formulation; $\mathbb{D}^{*}$ is the condensed form of the junction dynamic stiffness matrix - namely $\mathbb{D}=-\omega^{2} \mathbf{M}^{\mathrm{c}}+\mathbf{K}^{\mathrm{c}}\left(1+\mathrm{i} \eta^{\mathrm{c}}\right)^{5}-$ onto $\Gamma=\Gamma_{1} \cup \Gamma_{2}$ :

$$
\mathbb{D}^{*}=\mathbb{D}_{\mathrm{BB}}-\mathbb{D}_{\mathrm{BI}} \mathbb{D}_{\mathrm{II}}^{-1} \mathbb{D}_{\mathrm{IB}}
$$

where subscripts B / I refer to as interface / internal DOFs of the junction. Eq. (7) expresses a relationship between the displacements and forces applied to the

\footnotetext{
${ }^{5} \mathbf{M}^{\mathrm{c}}, \mathbf{K}^{\mathrm{c}}$ and $\eta^{\mathrm{c}}$ are respectively the mass matrix, the stiffness matrix and the loss factor of the junction.
} 
waveguides over the coupling interfaces. Introducing wave expansions of the form $\mathbf{u}_{i} \approx \widetilde{\boldsymbol{\Phi}}_{i} \widetilde{\mathbf{Q}}_{i}$ (cf. Eq. (4)) - with a reduced basis $\left\{\left(\widetilde{\boldsymbol{\Phi}}_{j}\right)_{i}\right\}_{j}$ of size $2 m_{i}\left(m_{i} \leq n_{i}\right)$ - into Eq. (7) also yields a relationship between the wave mode amplitudes to be considered as $[10,17]$ :

$$
\widetilde{\mathbf{A}}^{\text {ref }} \widetilde{\mathbf{Q}}^{\text {ref }}=-\widetilde{\mathbf{A}}^{\text {inc }} \widetilde{\mathbf{Q}}^{\text {inc }},
$$

where $\widetilde{\mathbf{Q}}^{\text {inc }}=\left[\widetilde{\mathbf{Q}}_{1}^{\text {inc } T} \widetilde{\mathbf{Q}}_{2}^{\text {inc } T}\right]^{T}$ and $\widetilde{\mathbf{Q}}^{\text {ref }}=\left[\widetilde{\mathbf{Q}}_{1}^{\text {ref } T} \widetilde{\mathbf{Q}}_{2}^{\text {ref } T}\right]^{T}$ are vectors of size $\left(\sum_{i} m_{i}\right) \times 1 ; \widetilde{\mathbf{A}}^{\text {ref }}$ and $\widetilde{\mathbf{A}}^{\text {inc }}$ are $\left(\sum_{i} n_{i}\right) \times\left(\sum_{i} m_{i}\right)$ matrices defined as

$$
\widetilde{\mathbf{A}}^{\text {ref }}=\mathbf{T}^{T} \mathbb{D}^{*} \mathbf{T} \widetilde{\mathbf{\Psi}}_{\mathrm{q}}^{\mathrm{ref}}+\widetilde{\mathbf{\Psi}}_{\mathrm{F}}^{\mathrm{ref}} \quad, \quad \widetilde{\mathbf{A}}^{\text {inc }}=\mathbf{T}^{T} \mathbb{D}^{*} \mathbf{T} \widetilde{\mathbf{\Psi}}_{\mathrm{q}}^{\text {inc }}+\widetilde{\mathbf{\Psi}}_{\mathrm{F}}^{\text {inc }},
$$

where $\widetilde{\boldsymbol{\Psi}}_{\mathrm{q}}^{\text {inc }}, \widetilde{\boldsymbol{\Psi}}_{\mathrm{q}}^{\text {ref }}, \widetilde{\boldsymbol{\Psi}}_{\mathrm{F}}^{\text {inc }}$ and $\widetilde{\boldsymbol{\Psi}}_{\mathrm{F}}^{\text {ref }}$ are matrices of same size $\left(\sum_{i} n_{i}\right) \times\left(\sum_{i} m_{i}\right)$, defined as:

$$
\begin{array}{ll}
\widetilde{\mathbf{\Psi}}_{\mathrm{q}}^{\text {inc }}=\left[\begin{array}{cc}
\mathcal{L}_{1}^{\mathrm{c}}\left(\widetilde{\boldsymbol{\Phi}}_{\mathrm{q}}^{\text {inc }}\right)_{1} & \mathbf{0} \\
\mathbf{0} & \mathcal{L}_{2}^{\mathrm{c}}\left(\widetilde{\boldsymbol{\Phi}}_{\mathrm{q}}^{\text {inc }}\right)_{2}
\end{array}\right] \quad, \quad \widetilde{\mathbf{\Psi}}_{\mathrm{q}}^{\text {ref }}=\left[\begin{array}{cc}
\mathcal{L}_{1}^{\mathrm{c}}\left(\widetilde{\boldsymbol{\Phi}}_{\mathrm{q}}^{\text {ref }}\right)_{1} & \mathbf{0} \\
\mathbf{0} & \mathcal{L}_{2}^{\mathrm{c}}\left(\widetilde{\boldsymbol{\Phi}}_{\mathrm{q}}^{\text {ref }}\right)_{2}
\end{array}\right], \\
\widetilde{\boldsymbol{\Psi}}_{\mathrm{F}}^{\text {inc }}=\left[\begin{array}{cc}
\mathcal{L}_{1}^{\mathrm{c}}\left(\widetilde{\boldsymbol{\Phi}}_{\mathrm{F}}^{\text {inc }}\right)_{1} & \mathbf{0} \\
\mathbf{0} & \mathcal{L}_{2}^{\mathrm{c}}\left(\widetilde{\boldsymbol{\Phi}}_{\mathrm{F}}^{\text {inc }}\right)_{2}
\end{array}\right] \quad, \quad \widetilde{\mathbf{\Psi}}_{\mathrm{F}}^{\text {ref }}=\left[\begin{array}{cc}
\mathcal{L}_{1}^{\mathrm{c}}\left(\widetilde{\boldsymbol{\Phi}}_{\mathrm{F}}^{\text {ref }}\right)_{1} & \mathbf{0} \\
\mathbf{0} & \mathcal{L}_{2}^{\mathrm{c}}\left(\widetilde{\boldsymbol{\Phi}}_{\mathrm{F}}^{\text {ref }}\right)_{2}
\end{array}\right] .
\end{array}
$$

Here, $\mathcal{L}_{i}^{\mathrm{c}}$ is a square $n_{i} \times n_{i}$ matrix that plays the role of expressing the displacements and forces of each waveguide $i$ in the coordinate system of the coupling junction $\left(x^{\mathrm{c}}, y^{\mathrm{c}}, z^{\mathrm{c}}\right)^{6}$.

The computation of Eq. (9) is addressed in the following:

Proposition 1. The matrix $\widetilde{\mathbf{A}}^{\text {ref }}$ is full column rank provided that $\operatorname{ran}\left(\mathbf{T} \widetilde{\mathbf{\Psi}}_{\mathrm{q}}^{\mathrm{ref}}\right) \perp \operatorname{null}\left(\mathbf{K}^{\mathrm{c} *}\right)$, where $\mathbf{K}^{\mathrm{c*}}$ is the condensed form of the junction stiffness matrix on $\Gamma$.

\footnotetext{
${ }^{6}$ The components of $\mathcal{L}_{i}^{\mathrm{c}}$ basically reflect the direction cosines of the local frame $\left(x_{i}, y_{i}, z_{i}\right)$, for the waveguide $i$, in the local frame $\left(x^{\mathrm{c}}, y^{\mathrm{c}}, z^{\mathrm{c}}\right)$.
} 
Proof. Let us proceed by contradiction and assume that $\widetilde{\mathbf{A}}^{\text {ref }}$ is not full column rank, i.e. its rank is different from the number of columns which is $\sum_{i} m_{i}$. This particularly means that there exists a family of modal amplitudes $\cup_{i}\left\{\left(\widetilde{Q}_{j}^{\text {ref }}\right)_{i}\right\}_{j=1, \ldots, m_{i}}$ with at least one non-zero component that satisfies

$$
\widetilde{\mathbf{A}}^{\mathrm{ref}} \widetilde{\mathbf{Q}}^{\mathrm{ref}}=\mathbf{0}
$$

Left multiplying Eq. (12) by $\left(\widetilde{\mathbf{\Psi}}_{\mathrm{q}}^{\text {ref }} \widetilde{\mathbf{Q}}^{\text {ref }}\right)^{H}$ (where $H$ denotes the conjugate transpose), considering Eq. (10) and using imaginary parts yield the following energy balance to be considered:

$$
\frac{\omega}{2} \operatorname{Im}\left(\left(\widetilde{\Psi}_{\mathrm{q}}^{\mathrm{ref}} \widetilde{\mathbf{Q}}^{\mathrm{ref}}\right)^{H} \mathbf{T}^{T} \mathbb{D}^{*} \mathbf{T}\left(\widetilde{\mathbf{\Psi}}_{\mathrm{q}}^{\mathrm{ref}} \widetilde{\mathbf{Q}}^{\mathrm{ref}}\right)\right)=\frac{\omega}{2} \operatorname{Im}\left(\left(\widetilde{\boldsymbol{\Psi}}_{\mathrm{q}}^{\mathrm{ref}} \widetilde{\mathbf{Q}}^{\mathrm{ref}}\right)^{H}\left(-\widetilde{\mathbf{\Psi}}_{\mathrm{F}}^{\mathrm{ref}} \widetilde{\mathbf{Q}}^{\mathrm{ref}}\right)\right)
$$

Physically, the left hand side term represents the power dissipated within the coupling junction (since structural damping is accounted for), while the right hand side term represents the opposite of the energy flow that leaves the coupling junction. It is well established from the law of Thermodynamics and energy-based formulations (SEA-like methods among others [21, 22]) that these two quantities are respectively positive and negative; as a result, the only way for $\widetilde{\mathbf{Q}}^{\text {ref }}$ to be the solution of Eq. (13) is that each of these two quantities is equal to zero. Regarding the left hand side term of Eq. (13), this particularly means that

$$
\operatorname{Im}\left(\left(\widetilde{\mathbf{\Psi}}_{\mathrm{q}}^{\mathrm{ref}} \widetilde{\mathbf{Q}}^{\mathrm{ref}}\right)^{H} \mathbf{T}^{T} \mathbb{D}^{*} \mathbf{T}\left(\widetilde{\boldsymbol{\Psi}}_{\mathrm{q}}^{\mathrm{ref}} \widetilde{\mathbf{Q}}^{\mathrm{ref}}\right)\right)=0
$$

Considering the condensation scheme (8), it can be proved without difficulty that Eq. (14) is in fact equivalent to

$$
\operatorname{Im}\left(\left(\mathbf{q}^{\mathrm{c}}\right)^{H} \mathbb{D} \mathbf{q}^{\mathbf{c}}\right)=0
$$


where $\mathbb{D}$ and $\mathbf{q}^{\mathrm{c}}$ are respectively the dynamic stiffness matrix and the displacement vector of the junction, the latter being defined as:

$$
\mathbf{q}^{\mathrm{c}}=\left[\begin{array}{c}
-\mathbb{D}_{\mathrm{II}}^{-1} \mathbb{D}_{\mathrm{IB}} \\
\mathbf{I}
\end{array}\right]\left(\mathbf{T} \widetilde{\mathbf{\Psi}}_{\mathrm{q}}^{\mathrm{ref}}\right) \widetilde{\mathbf{Q}}^{\mathrm{ref}} .
$$

Considering that dynamic stiffness matrix $\mathbb{D}$ is expressed as $-\omega^{2} \mathbf{M}^{\mathrm{c}}+\mathbf{K}^{\mathrm{c}}(1+$ $i \eta^{c}$ ), and considering that mass matrix $\mathbf{M}^{\mathrm{c}}$ and stiffness matrix $\mathbf{K}^{\mathrm{c}}$ are real symmetric (i.e. they are hermitian), it turns out that Eq. (15) is equivalent to:

$$
\left(\mathbf{q}^{\mathrm{c}}\right)^{H} \operatorname{Im}(\mathbb{D}) \mathbf{q}^{\mathrm{c}}=\eta^{\mathrm{c}}\left(\mathbf{q}^{\mathrm{c}}\right)^{H} \mathbf{K}^{\mathrm{c}} \mathbf{q}^{\mathrm{c}}=0
$$

Also note that $\mathbf{K}^{\mathrm{c}}$ is symmetric positive semi-definite [23], meaning that the only way for $\mathbf{q}^{\mathrm{c}}$ to be different from zero is that it is spanned by the null space of $\mathbf{K}^{\mathrm{c}}$, i.e. $\mathbf{K}^{\mathrm{c}} \mathbf{q}^{\mathrm{c}}=\mathbf{0}$. Using condensation procedure, this yields:

$$
\mathbf{K}^{\mathrm{c*}}\left(\mathbf{T} \widetilde{\boldsymbol{\Psi}}_{\mathrm{q}}^{\mathrm{ref}}\right) \widetilde{\mathbf{Q}}^{\mathrm{ref}}=\mathbf{0},
$$

where $\mathbf{K}^{\mathrm{c} *}=\mathbf{K}_{\mathrm{BB}}^{\mathrm{c}}-\mathbf{K}_{\mathrm{BI}}^{\mathrm{c}}\left(\mathbf{K}_{\mathrm{II}}^{\mathrm{c}}\right)^{-1} \mathbf{K}_{\mathrm{IB}}^{\mathrm{c}}$. Considering that $\operatorname{ran}\left(\mathbf{T} \widetilde{\mathbf{\Psi}}_{\mathrm{q}}^{\mathrm{ref}}\right) \perp \operatorname{null}\left(\mathbf{K}^{\mathrm{c} *}\right)$ (by assumption), it turns out necessarily that $\left(\mathbf{T} \widetilde{\mathbf{\Psi}}_{\mathrm{q}}^{\text {ref }}\right) \widetilde{\mathbf{Q}}^{\text {ref }}=\mathbf{0}$. Finally, considering that matrices $\mathbf{T}$ and $\widetilde{\mathbf{\Psi}}_{\mathrm{q}}^{\text {ref }}$ are full column rank ${ }^{7}$ yields $\widetilde{\mathbf{Q}}^{\text {ref }}=\mathbf{0}$, which is contradictory to the statement previously made that $\widetilde{\mathbf{A}}^{\text {ref }}$ is not full column rank.

Remark 1. The condition $\operatorname{ran}\left(\mathbf{T} \widetilde{\mathbf{\Psi}}_{\mathrm{q}}^{\text {ref }}\right) \perp \operatorname{null}\left(\mathbf{K}^{\mathrm{c} *}\right)$ is sufficient to prove the previous proposition. It could be violated at very low frequencies as rigid cross-section wave shapes are expected to interact with the rigid body motions of the junction;

\footnotetext{
${ }^{7}$ This can be proved as: (i) the rank of $\mathbf{T}$ represents the number of DOFs contained over the two waveguide cross-sections; (ii) the wave mode matrices $\left(\widetilde{\mathbf{\Phi}}_{\mathrm{q}}^{\text {ref }}\right)_{1}$ and $\left(\widetilde{\mathbf{\Phi}}_{\mathrm{q}}^{\text {ref }}\right)_{2}$ are full column rank (a formal proof is brought in ref. [3], Proposition 1).
} 
this remains possible provided the waveguides are positioned in such a manner that those rigid body motions are possible. However, as the frequency increases, the fact that wave mode shapes are subjected to local deformations prevents such an issue.

Proposition 1 means that the left pseudo-inverse of $\widetilde{\mathbf{A}}^{\text {ref }}$ - namely $\left(\widetilde{\mathbf{A}}^{\text {ref }}\right)^{+}$ - can be computed as $\left[\left(\widetilde{\mathbf{A}}^{\text {ref }}\right)^{H} \widetilde{\mathbf{A}}^{\text {ref }}\right]^{-1}\left(\widetilde{\mathbf{A}}^{\text {ref }}\right)^{H}$ [24]. It also means that there exists a unique solution $\widetilde{\mathbf{Q}}^{\text {ref }}$ of Eq. (9) that satisfies the following least squares (LS) problem:

$$
\underset{\widetilde{\mathbf{Q}}^{\text {ref }}}{\min }\left\|\widetilde{\mathbf{A}}^{\text {ref }} \widetilde{\mathbf{Q}}^{\text {ref }}+\widetilde{\mathbf{A}}^{\text {inc }} \widetilde{\mathbf{Q}}^{\text {inc }}\right\|_{2} .
$$

This solution is thus given by [24]:

$$
\widetilde{\mathbf{Q}}^{\mathrm{ref}}=-\left(\widetilde{\mathbf{A}}^{\mathrm{ref}}\right)^{+} \widetilde{\mathbf{A}}^{\text {inc }} \widetilde{\mathbf{Q}}^{\text {inc }} .
$$

This provides a unique way to determine the amplitudes of the reflected modes from those of the incident modes. This validates the formulation proposed in previous works $[10,17]$, i.e.:

$$
\widetilde{\mathbf{Q}}^{\text {ref }}=\widetilde{\mathbb{C}} \widetilde{\mathbf{Q}}^{\text {inc }} \text { where } \widetilde{\mathbb{C}}=-\left(\widetilde{\mathbf{A}}^{\text {ref }}\right)^{+} \widetilde{\mathbf{A}}^{\text {inc }} .
$$

The matrix $\widetilde{\mathbb{C}}$ physically expresses the reflection and transmission coefficients of the wave modes across the coupling junction. It can be readily decomposed so as to emphasize the dependency among the wave modes between waveguides:

$$
\left[\begin{array}{c}
\widetilde{\mathbf{Q}}_{1}^{\text {ref }} \\
\widetilde{\mathbf{Q}}_{2}^{\text {ref }}
\end{array}\right]=\left[\begin{array}{ll}
\widetilde{\mathbb{C}}_{11} & \widetilde{\mathbb{C}}_{12} \\
\widetilde{\mathbb{C}}_{21} & \widetilde{\mathbb{C}}_{22}
\end{array}\right]\left[\begin{array}{l}
\widetilde{\mathbf{Q}}_{1}^{\text {inc }} \\
\widetilde{\mathbf{Q}}_{2}^{\text {inc }}
\end{array}\right] .
$$

Remark 2. The components of $\widetilde{\mathbb{C}}$ are influenced by the way the vectors $\left\{\left(\widetilde{\boldsymbol{\Phi}}_{j}\right)_{i}\right\}_{j}$ are scaled. Choosing different strategies for scaling these vectors can yield largely 
disparate results for the components of $\widetilde{\mathbb{C}}$. Such an issue is not encountered in analytical approaches involving cross-sections with one or two DOFs, since wave shapes are quite simple in this case. This constitutes a challenge for plotting the reflection / transmission coefficients and interpreting their physical behavior in the frequency domain, e.g. by means of comparisons with analytical results. Scaling each wave shape $\left(\widetilde{\boldsymbol{\Phi}}_{j}\right)_{i}$ so that the maximum value of their displacement components is set to unity seems to constitute an adequate solution. It is worth noting that such an issue does not affect the computation of the forced responses as scaling effects of $\left\{\left(\widetilde{\boldsymbol{\Phi}}_{j}\right)_{i}\right\}_{j}$ disappear through the wave mode expansion procedure.

\subsection{Forced response computation}

The problem of predicting the frequency response of the coupled system depicted in Figure 1 is addressed. The system involves two straight waveguides 1 and 2, composed respectively of $N_{1}$ and $N_{2}$ substructures, connected through a common junction over one of their boundaries. Considering the other boundaries, waveguides 1 and 2 are submitted to prescribed forces $\mathbf{F}_{0}$ and displacements $\mathbf{q}_{0}$, respectively. Such a problem involving Neumann and Dirichlet boundary conditions has been addressed in [3]. In brief, the boundary conditions are addressed by means of wave mode expansions of the form (4). In particular, the coupling conditions through the junction are addressed by means of Eq. (21). A specific scaling procedure for treating matrix structures with largely disparate terms - between the components of $\widetilde{\boldsymbol{\mu}}^{-N}$ and $\widetilde{\boldsymbol{\mu}}^{N}$ (cf. Eq. (5)) and between the components of $\widetilde{\boldsymbol{\Phi}}_{\mathrm{q}}$ and 
$\widetilde{\Phi}_{\mathrm{F}}-$ is used. This yields the following well-conditioned problem to be computed:

$$
\begin{aligned}
& {\left[\begin{array}{cc|cc}
\mathbf{I} & \left(\widetilde{\boldsymbol{\Phi}}_{\mathrm{F}}^{\text {inc }}\right)_{1}^{+}\left(\widetilde{\boldsymbol{\Phi}}_{\mathrm{F}}^{\text {ref }}\right)_{1} \widetilde{\boldsymbol{\mu}}_{1}^{N_{1}} & \mathbf{0} & \mathbf{0} \\
-\widetilde{\mathbb{C}}_{11} \widetilde{\boldsymbol{\mu}}_{1}^{N_{1}} & \mathbf{I} & \mathbf{0} & -\widetilde{\mathbb{C}}_{12} \widetilde{\boldsymbol{\mu}}_{2}^{N_{2}} \\
\hline-\widetilde{\mathbb{C}}_{21} \widetilde{\boldsymbol{\mu}}_{1}^{N_{1}} & \mathbf{0} & \mathbf{I} & -\widetilde{\mathbb{C}}_{22} \widetilde{\boldsymbol{\mu}}_{2}^{N_{2}} \\
\mathbf{0} & \mathbf{0} & \left(\widetilde{\boldsymbol{\Phi}}_{\mathrm{q}}^{\text {inc }}\right)_{2}^{+}\left(\widetilde{\boldsymbol{\Phi}}_{\mathrm{q}}^{\text {ref }}\right)_{2} \widetilde{\boldsymbol{\mu}}_{2}^{N_{2}} & \mathbf{I}
\end{array}\right]} \\
& \times\left[\begin{array}{cc|cc}
\mathbf{I} & \mathbf{0} & \mathbf{0} & \mathbf{0} \\
\mathbf{0} & \widetilde{\boldsymbol{\mu}}_{1}^{-N_{1}} & \mathbf{0} & \mathbf{0} \\
\hline \mathbf{0} & \mathbf{0} & \widetilde{\boldsymbol{\mu}}_{2}^{-N_{2}} & \mathbf{0} \\
\mathbf{0} & \mathbf{0} & \mathbf{0} & \mathbf{I}
\end{array}\right]\left[\begin{array}{c}
\widetilde{\mathbf{Q}}_{1}^{\text {inc }(1)} \\
\widetilde{\mathbf{Q}}_{1}^{\text {ref }(1)} \\
\widetilde{\mathbf{Q}}_{2}^{\text {ref }(1)} \\
\widetilde{\mathbf{Q}}_{2}^{\text {inc }(1)}
\end{array}\right]=\left[\begin{array}{c}
-\left(\widetilde{\boldsymbol{\Phi}}_{\mathrm{F}}^{\text {inc }}\right)_{1}^{+} \mathbf{F}_{0} \\
\mathbf{0} \\
\hline \mathbf{0} \\
\left(\widetilde{\boldsymbol{\Phi}}_{\mathrm{q}}^{\text {inc }}\right)_{2}^{+} \mathbf{q}_{0}
\end{array}\right],
\end{aligned}
$$

where the first matrix (whose size is $\left.2\left(\sum_{i} m_{i}\right) \times 2\left(\sum_{i} m_{i}\right)\right)$ is expected to be well conditioned [3], while the second matrix is diagonal and can be inverted without difficulty. Solving Eq. (23) provides the modal amplitudes $\left\{\widetilde{\mathbf{Q}}_{1}^{\text {inc }(1)}, \widetilde{\mathbf{Q}}_{1}^{\text {ref }(1)}\right\}$ and $\left\{\widetilde{\mathbf{Q}}_{2}^{\text {inc(1) }}, \widetilde{\mathbf{Q}}_{2}^{\text {ref(1) }}\right\}$ at the ends of waveguides 1 and 2 where forces and displacements are respectively prescribed. The spatial distribution of the modal amplitudes along each waveguide follows from Eq. (5), while the spatial distribution of the kinematic variables (i.e. displacements and internal forces) follows from Eq. (4).

Remark 3. Within the WFE framework, the strategy used for computing the forced response of coupled systems can be summarized through the following steps (to be considered at each frequency):

1. Computation of wave modes by means of an eigenproblem [3] of size $2 n_{i} \times$ $2 n_{i}$ (to be done for each waveguide $i$, if different);

2. Computation of $\mathbb{D}^{*}$ by means of Eq. (8), where the inverse of a $n_{\mathrm{I}}^{c} \times n_{\mathrm{I}}^{c}$ matrix $\mathbb{D}_{\mathrm{II}}$ is required $\left(n_{\mathrm{I}}^{\mathrm{c}}\right.$ denotes the number of internal DOFs of the junction); 
3. Computation of $\mathbb{C}$ by means of Eq. (21), where the pseudo-inverse of the $\left(\sum_{i} n_{i}\right) \times\left(\sum_{i} m_{i}\right)$ matrix $\widetilde{\mathbf{A}}^{\text {ref }}$ is required;

4. Computation of Eq. (23), where the inverse of a $2\left(\sum_{i} m_{i}\right) \times 2\left(\sum_{i} m_{i}\right)$ matrix is required.

The sizes of the matrix subproblems involved in these steps do not depend on the number of substructures considered for discretizing the waveguides. This yields the CPU times to be considerably lowered compared to what is required by the standard FE method when computing the full numerical model. Indeed, following the classic FE framework yields the size of the full matrix problem to be rather $\left[n^{\mathrm{c}}+\sum_{i} n_{i}\left(N_{i}+1\right)\right] \times\left[n^{\mathrm{c}}+\sum_{i} n_{i}\left(N_{i}+1\right)\right]$ where $N_{i}$ is the number of substructures used for each waveguide $i$ and $n^{\mathrm{c}}$ is the number of junction DOFs. It is worth noting that steps 1-3 are to be addressed once and for all, whatever the lengths and excitation sources of the waveguides change. This emphasizes a relative flexibility of the WFE approach in what only the small matrix problem (23) is to be re-computed in case of such changes.

The forced response of the coupled system depicted in Figure 1 is computed in the next subsection. The issue consists in investigating the relevance of the WFE method further on in the frequency domain (compared to the results exposed in ref. [3]), i.e. when the junction undergoes resonances. The reflection / transmission coefficients for wave modes and energy flows, across the junction, are also investigated. 


\subsection{Numerical results}

\subsubsection{Forced response}

The wave-based matrix formulation (23) is applied for computing the forced response of the coupled system depicted in Figure 1, where two straight waveguides with similar rectangular cross-sections are involved. The waveguides are coupled "transversally" by means of an elastic junction which represents a quarter of torus: the main axes of the two waveguides - say axes $x_{1}$ and $x_{2}-$ are perpendicular so that coupling among wave modes of different natures (say for instance, flexural and torsional) is likely to occur. Apart from the coupling conditions, considering other boundaries, waveguides 2 and 1 are respectively clamped (i.e. $\mathbf{q}_{0}=\mathbf{0}$ ) and subjected to a uniform transverse force field that reflects vector $\mathbf{F}_{0}$. The two waveguides, as well as the coupling junction, exhibit the same material characteristics: Young's modulus $E=3.2 \times 10^{9} \mathrm{~Pa}$, density $\rho=1180 \mathrm{~kg} . \mathrm{m}^{-3}$, Poisson's ratio $\nu=0.39$, loss factor $\eta=0.01$. The two waveguides have the same crosssectional area $h_{y} \times h_{z}=0.2 \mathrm{~m} \times 0.15 \mathrm{~m}$, while their respective lengths are $L_{1}=2$ $\mathrm{m}$ and $L_{2}=1.5 \mathrm{~m}$. The quarter of torus has an internal radius of curvature of $R^{\mathrm{c}}=0.05 \mathrm{~m}$ and a cross-section similar to those of the connected waveguides. The waveguides 1 and 2 are discretized respectively by means of $N_{1}=100$ and $N_{2}=75$ similar substructures, each of these being composed of $4 \times 3$ linear brick elements and having a length $d=0.02 \mathrm{~m}$ (see Figure 2). Linear brick elements are also used for discretizing the junction. Each coupling interface is interpolated with $5 \times 4$ elements. In this case, the mesh compatibility over coupling interfaces is not verified. Such a dissimilarity is invoked here to address the wave propagation along waveguides apart from the internal dynamics of the coupling junction.

The total number of DOFs used for discretizing the coupling junction is 810 , while $2 \times 60$ DOFs are used for discretizing each substructure. It is readily ver- 
ified that the full FE model of the coupled system involves $810+60\left(N_{1}+1\right)+$ $60\left(N_{2}+1\right)=8775$ DOFs. On the other hand, the WFE formulation (23) uses two reduced wave mode bases of respective sizes $2 m_{1}$ and $2 m_{2}$ (with $m_{1}, m_{2} \leq 60$ ) for describing the waveguides; in the present case, $m_{1}=m_{2}=m$. This yields the size of the wave-based matrix problem to be $4 m \times 4 m$ with $4 m \leq 240$, which is much less than the full FE model whose size is $8775 \times 8775$.

The forced response of the coupled system is addressed over the frequency band $\mathcal{B}_{f}=[10 \mathrm{~Hz}, 5000 \mathrm{~Hz}]$ which appears to be the same as the one involved in previous work [3]. Nonetheless, the local dynamics are expected to be more complex in the present case, especially in terms of junction resonances, as quite soft materials are considered. Eq. (23) is computed for providing the WFE displacement solution, by means of Eq. (4). For each waveguide $i$, the wave shape basis $\left\{\left(\widetilde{\boldsymbol{\Phi}}_{j}\right)_{i}\right\}_{j}$ is supposed to include the classic LF modes as well as additional MF modes whose contribution can be significant. Some of these shapes are depicted in Figure 3 at $2500 \mathrm{~Hz}$. They refer to as the classic LF flexural, torsional, longitudinal and shearing modes with a non-uniform spatial behavior ${ }^{8}$, and some MF higher order modes with an oscillating spatial dynamics for capturing the cross-section resonances. The solutions provided by WFE (cf. Eq. (23)) with wave bases of different sizes are compared with the standard FE solution, i.e. when the full FE model is computed. The results are shown in Figure 5, where the transverse displacement of waveguide 1 (at one corner of its excited cross-section) is plotted for 500 discrete frequencies. The reference solution provided by FE highlights a relative complexity of the frequency behavior above $2500 \mathrm{~Hz}$, i.e. when the local dynamics of the junction are solicited. Below this threshold, the behavior of the

\footnotetext{
${ }^{8}$ It is worth recalling that the rigid body assumption for the cross-section breaks down at high frequencies.
} 
coupled system is mainly driven by the resonances of the waveguides, while the response of the junction is mainly static. In this range, the WFE method works fine with a few modes (see Figure 5(a)). At higher frequencies, the size of the wave bases has to be enlarged to ensure the convergence of the method. Small errors still subsist with $m=40$ incident / reflected modes, around and above $3000 \mathrm{~Hz}$. The convergence is finally achieved when $m=50$ incident / reflected modes are used.

\section{Figure 5}

\subsubsection{Reflection / transmission coefficients of wave modes and energy flows}

As mentioned previously, the computation of the forced response is directly linked to that of the matrix $\mathbb{C}$, whose components are the reflection and transmission coefficients of wave modes across the coupling junction. As mentioned, the way these coefficients are plotted strongly depends on how the wave mode shapes are scaled. Choosing an appropriate strategy to scale the wave modes in a "continuous way" along the frequency domain (e.g. by means of the maximum value of the wave displacement components) can yield regular curves to be drawn. Several components of $\mathbb{C}$ are computed in this sense, when the size of the wave basis is $2 m=2 \times 50$ for each waveguide ${ }^{9}$. For example, the real part of the reflection / transmission coefficients among the flexural mode (Figure 3(a)), the torsional mode (Figure 3(b)) and a given MF mode (Figure 3(e)) are presented in Figure 6. As expected (see above), the flexural and torsional wave motions appear correlated through the coupling junction. Mode conversions also occur between the flexural / torsional modes and the MF mode around $3000 \mathrm{~Hz}$, i.e. where the local dynamics of the junction are involved. The fact that the shape of this MF mode (see Figure 3(e)) appears as a type of high order torsional wave motion could partly explain

\footnotetext{
${ }^{9}$ The relevance of such a size with $m=50$ has been emphasized above.
} 
why this mode is coupled with the others.

\section{Figure 6}

The way the mode conversion operates is usually examined from the point of view of energy flows (i.e. powers). The derivation of power reflection / transmission coefficients is detailed in Appendix A. These are shown in Figures 7 and 8 for the incident flexural and torsional modes (a) and (b) (cf. Figure 3). Reflection / transmission between these modes and shearing / MF modes (d) and (e) (cf. Figure 3 ) is particularly addressed. The flexural and torsional modes appear strongly coupled each other below $3300 \mathrm{~Hz}$ when shearing / MF modes are mainly evanescent. This appears coherent in the sense that evanescent modes do not convey energy [25]. At higher frequencies, the shearing / MF wave modes become propagating. Coupling phenomena appear quite complex as energy exchanges with these modes occur. Particularly, retaining MF modes in the wave bases for computing the WFE displacement solutions becomes relevant. It is shown that power reflection / transmission coefficients exhibit a frequency behavior which is oscillating, i.e. driven in part by the local dynamics of the junction.

\section{Figure 7}

\section{Figure 8}

\section{CMS-based approach}

\subsection{Introduction}

The relevance of the WFE formulation has been highlighted for computing the forced response of coupled systems. The local dynamics of the coupling junction have been addressed using FE analysis and a condensed form of the dynamic 
stiffness matrix. Another way to assess the behavior of the junction is to consider component mode synthesis (CMS) procedures, i.e. using a reduced family of elastic modes as a representation basis. The motivation is to reduce the CPU times further and to quantify the impact of these junction modes onto the response of the global system. Such an analysis is commonly performed in the framework of uncertainty propagation to address small perturbations at the scale of component modes [16]. The fact that a few junction modes are dealt with is the key idea to address such an analysis with reasonable computational times, e.g. when performing Monte Carlo simulations (MCS). This suggests to select among all the junction modes those which effectively contribute to the system forced response. This issue is investigated hereafter. Among all the variety of CMS procedures, the CraigBampton (CB) method will be investigated specifically. Indeed, the relevance of such a procedure has been widely proved for treating coupled problems involving FE substructures with a small number of elastic modes $[26,16]$.

\subsection{CMS framework}

The CB method is used for addressing the junction dynamics. For the sake of clarity, the case of two waveguides connected with a single junction is considered (cf. Figure 1). The basics of the CB method are recalled below:

Let $\Gamma=\Gamma_{1} \cup \Gamma_{2}$ denotes the interface between the junction and the waveguides. The junction displacements are expressed in terms of a reduced family of fixed-interface elastic modes $\left\{\left(\widetilde{\mathbf{X}}_{\mathrm{e} 1}\right)_{j}\right\}_{j=1, \ldots, m^{\mathrm{c}}}\left(m^{\mathrm{c}}\right.$ being the number of retained modes) combined with constraint modes $\left\{\left(\mathbf{X}_{\mathrm{st}}\right)_{j}\right\}_{j=1, \ldots, n_{\mathrm{B}}^{\mathrm{c}}}\left(n_{\mathrm{B}}^{\mathrm{c}}\right.$ being the number of DOFs contained over $\Gamma)^{10}$. This yields the displacements of internal and in-

\footnotetext{
${ }^{10}$ The constraint modes express the static response of the system in terms of interface displace-
} 
terface DOFs - denoted as $\mathbf{q}_{\mathrm{I}}^{\mathrm{c}}$ and $\mathbf{q}_{\mathrm{B}}^{\mathrm{c}}=\left.\mathbf{q}^{\mathrm{c}}\right|_{\Gamma}$, respectively - to be approximated as

$$
\left[\begin{array}{c}
\mathbf{q}_{\mathrm{I}}^{\mathrm{c}} \\
\mathbf{q}_{\mathrm{B}}^{\mathrm{c}}
\end{array}\right] \approx\left[\begin{array}{cc}
\widetilde{\mathbf{X}}_{\mathrm{el}} & \mathbf{X}_{\mathrm{st}} \\
\mathbf{0} & \mathbf{I}
\end{array}\right]\left[\begin{array}{c}
\widetilde{\boldsymbol{\alpha}} \\
\mathbf{q}_{\mathrm{B}}^{\mathrm{c}}
\end{array}\right],
$$

where $\widetilde{\boldsymbol{\alpha}}$ is the vector of modal amplitudes; $\widetilde{\mathbf{X}}_{\mathrm{el}}$ is the $n_{\mathrm{I}}^{\mathrm{c}} \times m^{\mathrm{c}}$ matrix of elastic modes, while $\mathbf{X}_{\mathrm{st}}$ is the $n_{\mathrm{I}}^{\mathrm{c}} \times n_{\mathrm{B}}^{\mathrm{c}}$ matrix of constraint modes:

$$
\mathbf{X}_{\mathrm{st}}=-\left(\mathbf{K}_{\mathrm{II}}^{\mathrm{c}}\right)^{-1} \mathbf{K}_{\mathrm{IB}}^{\mathrm{c}}
$$

Here, $\mathbf{K}^{\mathrm{c}}$ refers to as the junction stiffness matrix. Invoking the junction dynamic stiffness matrix $\mathbb{D}$ (cf. subsection 2.2) with the basis of elastic and constraint modes leads to [16]:

$$
\left[\begin{array}{cc}
\widetilde{\mathbb{D}}_{\mathrm{e} 1-\mathrm{el}} & \widetilde{\mathbb{D}}_{\mathrm{el}-\mathrm{st}} \\
\widetilde{\mathbb{D}}_{\mathrm{e} 1-\mathrm{st}}^{T} & \mathbb{D}_{\mathrm{st}-\mathrm{st}}
\end{array}\right]\left[\begin{array}{c}
\widetilde{\boldsymbol{\alpha}} \\
\mathbf{q}_{\mathrm{B}}^{\mathrm{c}}
\end{array}\right] \approx\left[\begin{array}{c}
\mathbf{0} \\
\mathbf{F}_{\mathrm{B}}^{\mathrm{c}}
\end{array}\right],
$$

where $\mathbf{F}_{\mathrm{B}}^{\mathrm{c}}=\left.\mathbf{F}^{\mathrm{c}}\right|_{\Gamma}$, while $\widetilde{\mathbb{D}}_{\mathrm{el}-\mathrm{el}}, \widetilde{\mathbb{D}}_{\mathrm{el}-\mathrm{st}}$ and $\mathbb{D}_{\mathrm{st}-\mathrm{st}}$ are expressed as:

$$
\begin{aligned}
& \widetilde{\mathbb{D}}_{\mathrm{el}-\mathrm{el}}=\operatorname{diag}\left\{\widetilde{\gamma}_{j}\left(-\omega^{2}+\widetilde{\omega}_{j}^{2}\left(1+\mathrm{i} \eta^{\mathrm{c}}\right)\right)\right\}_{j=1, \ldots m^{\mathrm{c}}}, \\
& \widetilde{\mathbb{D}}_{\mathrm{el}-\mathrm{st}}=-\omega^{2} \widetilde{\mathbf{X}}_{\mathrm{el}}^{T}\left(\mathbf{M}_{\mathrm{II}}^{\mathrm{c}} \mathbf{X}_{\mathrm{st}}+\mathbf{M}_{\mathrm{IB}}^{\mathrm{c}}\right), \\
& \mathbb{D}_{\mathrm{st}-\mathrm{st}}=-\omega^{2}\left(\mathbf{X}_{\mathrm{st}}^{T} \mathbf{M}_{\mathrm{II}}^{\mathrm{c}} \mathbf{X}_{\mathrm{st}}+\mathbf{M}_{\mathrm{BI}}^{\mathrm{c}} \mathbf{X}_{\mathrm{st}}+\mathbf{X}_{\mathrm{st}}^{T} \mathbf{M}_{\mathrm{IB}}^{\mathrm{c}}+\mathbf{M}_{\mathrm{BB}}^{\mathrm{c}}\right)+\left(1+i \eta^{\mathrm{c}}\right)\left(\mathbf{K}_{\mathrm{BI}}^{\mathrm{c}} \mathbf{X}_{\mathrm{st}}+\mathbf{K}_{\mathrm{BB}}^{\mathrm{c}}\right) .
\end{aligned}
$$

Here, $\widetilde{\omega}_{j}$ and $\widetilde{\gamma}_{j}$ represent the eigenpulsation and the modal mass of each elastic mode $j$, respectively: the modal mass is readily written as $\widetilde{\gamma}_{j}=\left(\widetilde{\mathbf{X}}_{\mathrm{el}}\right)_{j}^{T} \mathbf{M}_{\mathrm{II}}^{\mathrm{c}}\left(\widetilde{\mathbf{X}}_{\mathrm{el}}\right)_{j}$ and can be set to unity provided that eigenvectors $\left\{\left(\widetilde{\mathbf{X}}_{\mathrm{el}}\right)_{j}\right\}_{j}$ are normalized appropriately [27]. Condensing the dynamic stiffness matrix - approximated as in Eq. (26) - onto the interface $\Gamma$ finally results in

$$
\widetilde{\mathbb{D}}^{*} \mathbf{q}_{\mathrm{B}}^{\mathrm{c}} \approx \mathbf{F}_{\mathrm{B}}^{\mathrm{c}}
$$

ments. 
where $\widetilde{\mathbb{D}}^{*}$ is constructed as:

$$
\widetilde{\mathbb{D}}^{*}=\mathbb{D}_{\text {st-st }}-\widetilde{\mathbb{D}}_{\mathrm{el}-\mathrm{st}}^{T} \widetilde{\mathbb{D}}_{\mathrm{el-el}}^{-1} \widetilde{\mathbb{D}}_{\mathrm{el}-\mathrm{st}} .
$$

Eq. (28) is quite similar to what was used to derive the mesh tying formulation (7), except that $\mathbb{D}^{*}$ has been replaced by $\widetilde{\mathbb{D}}^{*}$. The latter is expressed in terms of the modal parameters of the junction, e.g. eigenvectors $\left\{\left(\widetilde{\mathbf{X}}_{\mathrm{el}}\right)_{j}\right\}_{j}$ and eigenpulsations $\left\{\widetilde{\omega}_{j}\right\}_{j}$. This leads the way in formulating the matrix $\mathbb{C}$ and expressing the dynamic response of the global system in terms of these modal parameters.

\subsection{Selection of contributing modes}

Eq. (28) uses a reduced family of eigenvectors $\left\{\left(\widetilde{\mathbf{X}}_{\mathrm{el}}\right)_{j}\right\}_{j=1, \ldots, m^{c}}$ for approximating the junction dynamic stiffness matrix as $\mathbb{D}^{*} \approx \widetilde{\mathbb{D}}^{*}$. This reduced family is extracted from the full family of junction modes - namely $\left\{\left(\mathbf{X}_{\mathrm{el}}\right)_{j}\right\}_{j=1, \ldots, n_{\mathrm{I}}^{\text {c }}}$ $\left(n_{\mathrm{I}}^{c} \geq m^{c}\right)$ - whose size $n_{\mathrm{I}}^{\mathrm{c}}$ relates the number of junction internal DOFs. Rigorously, invoking the full family $\left\{\left(\mathbf{X}_{\mathrm{el}}\right)_{j}\right\}_{j}$ instead of $\left\{\left(\widetilde{\mathbf{X}}_{\mathrm{el}}\right)_{j}\right\}_{j}$ enables $\widetilde{\mathbb{D}}^{*}$ to be equal to $\mathbb{D}^{* 11}$. According to Eqs. (29) and (27), this results as:

$$
\mathbb{D}^{*}=\mathbb{D}_{\mathrm{st}-\mathrm{st}}-\sum_{j=1}^{n_{\mathrm{I}}^{\mathrm{I}}}\left(\frac{\omega^{4}}{-\omega^{2}+\omega_{j}^{2}\left(1+\mathrm{i} \eta^{\mathrm{c}}\right)}\left(\mathbf{B}^{T}\left(\mathbf{X}_{\mathrm{el}}\right)_{j}\right)\left(\mathbf{B}^{T}\left(\mathbf{X}_{\mathrm{el}}\right)_{j}\right)^{T}\right),
$$

where $\mathbf{B}=\mathbf{M}_{\mathrm{II}}^{\mathrm{c}} \mathbf{X}_{\mathrm{st}}+\mathbf{M}_{\mathrm{IB}}^{\mathrm{c}}$, while assumption is made that $\widetilde{\gamma}_{j}=1 \forall j$ (see previous subsection). In brief, the fact that one mode $j$ is neglected in Eq. (30) yields the matrix $\mathbb{D}^{*}$ to be perturbed as $\mathbb{D}^{*}+\Delta_{j} \mathbb{D}^{*}$, where

$$
\Delta_{j} \mathbb{D}^{*}=\frac{\omega^{4}}{-\omega^{2}+\omega_{j}^{2}\left(1+\mathrm{i} \eta^{\mathrm{c}}\right)}\left(\mathbf{B}^{T}\left(\mathbf{X}_{\mathrm{el}}\right)_{j}\right)\left(\mathbf{B}^{T}\left(\mathbf{X}_{\mathrm{el}}\right)_{j}\right)^{T} \quad j=1, \ldots, n_{\mathrm{I}}^{\mathrm{c}} .
$$

\footnotetext{
${ }^{11}$ This can be proved as the full family exhibits a dimension $n_{\mathrm{I}}^{\mathrm{c}}$ equal to the rank of the matrix $\mathbb{D}_{\mathrm{II}}$.
} 
The key idea here is to quantify the impact of perturbating $\mathbb{D}^{*}$ as $\mathbb{D}^{*}+\Delta_{j} \mathbb{D}^{*}$ onto the forced response of the coupled system. Within the WFE framework, this suggests to quantify the resulting perturbation onto the solution of the LS problem (19), i.e. for determining the amplitudes of reflected waves $\widetilde{\mathbf{Q}}^{\text {ref }}$ from a known state of incident waves $\widetilde{\mathbf{Q}}^{\text {inc }}$. A norm-wise procedure, by means of any consistent norm $\|$.$\| [24], appears suitable for addressing this task [13, 14, 15]. This sug-$ gests to compute the norm-wise relative error $\left\|\Delta_{j} \widetilde{\mathbf{Q}}^{\text {ref }}\right\| /\left\|\widetilde{\mathbf{Q}}^{\text {ref }}\right\|$ induced while computing Eq. (21) with $\mathbb{D}^{*}+\Delta_{j} \mathbb{D}^{*}$.

Proposition 2. Suppose that $\|$.$\| is a consistent norm and that \left\|\widetilde{\mathbf{Q}}^{\text {ref }}\right\| \neq 0$ and $\|\widetilde{\mathbb{C}}\| \neq 0$; also suppose that $\Delta_{j} \widetilde{\mathbf{Q}}^{\text {inc }}=\mathbf{0}$. Then the relative error $\left\|\Delta_{j} \widetilde{\mathbf{Q}}^{\text {ref }}\right\| /\left\|\widetilde{\mathbf{Q}}^{\text {ref }}\right\|$ can be bounded as

$$
\frac{\left\|\Delta_{j} \widetilde{\mathbf{Q}}^{\text {ref }}\right\|}{\left\|\widetilde{\mathbf{Q}}^{\text {ref }}\right\|} \leq \frac{\left\|\Delta_{j} \widetilde{\mathbb{C}}\right\|}{\|\widetilde{\mathbb{C}}\|} \quad j=1, \ldots, n_{\mathrm{I}}^{\mathrm{c}},
$$

provided $\widetilde{\mathbf{Q}}^{\text {inc }}$ is unitary and such that $\left\|\widetilde{\mathbb{C}} \widetilde{\mathbf{Q}}^{\text {inc }}\right\|=\|\widetilde{\mathbb{C}}\|$.

Proof. The proof is straightforward from Eq. (21), considering $\left\|\widetilde{\mathbf{Q}}^{\text {ref }}\right\|=\left\|\widetilde{\mathbb{C}} \widetilde{\mathbf{Q}}^{\text {inc }}\right\|=$ $\|\widetilde{\mathbb{C}}\|$ and $\Delta_{j} \widetilde{\mathbf{Q}}^{\text {ref }}=\left(\Delta_{j} \widetilde{\mathbb{C}}\right) \widetilde{\mathbf{Q}}^{\text {inc }}\left(\right.$ since $\Delta_{j} \widetilde{\mathbf{Q}}^{\text {inc }}=\mathbf{0}$ ): this yields $\left\|\Delta_{j} \widetilde{\mathbf{Q}}^{\text {ref }}\right\|=$ $\left\|\left(\Delta_{j} \widetilde{\mathbb{C}}\right) \widetilde{\mathbf{Q}}^{\text {inc }}\right\| \leq\left\|\Delta_{j} \widetilde{\mathbb{C}}\right\|\left\|\widetilde{\mathbf{Q}}^{\text {inc }}\right\|=\left\|\Delta_{j} \widetilde{\mathbb{C}}\right\|$, because the norm $\|$.$\| is consistent$ and $\widetilde{\mathbf{Q}}^{\text {inc }}$ is unitary.

Proposition 2 addresses a norm-wise bound of $\left\|\Delta_{j} \widetilde{\mathbf{Q}}^{\text {ref }}\right\| /\left\|\widetilde{\mathbf{Q}}^{\text {ref }}\right\|$ by means of $\left\|\Delta_{j} \widetilde{\mathbb{C}}\right\| / \| \widetilde{\mathbb{C}}||$ only, without computing $\widetilde{\mathbf{Q}}^{\text {inc }}$ by means of Eq. (23) (i.e. without invoking the excitation sources). This yields the issue of large CPU times to be circumvented while considering different loading cases. It is worth noting that assumptions $\left\|\widetilde{\mathbf{Q}}^{\text {inc }}\right\|=1$ and $\left\|\widetilde{\mathbb{C}} \widetilde{\mathbf{Q}}^{\text {inc }}\right\|=\|\widetilde{\mathbb{C}}\|$ are not restrictive. They can be verified a priori for any kind of excitation source and wave shape fields over the cross-sections, provided the magnitudes of the latter are correctly scaled. 
Remark 4. Formulating bounds for $\left\|\Delta_{j} \widetilde{\mathbf{Q}}^{\text {ref }}\right\| /\left\|\widetilde{\mathbf{Q}}^{\text {ref }}\right\|$ addresses perturbation theories for LS problems of the general form $\min _{\mathbf{x}}\|\mathbf{A x}-\mathbf{b}\|_{2}(\mathbf{A}, \mathbf{x}$ and $\mathbf{b}$ being understood as $\widetilde{\mathbf{A}}^{\text {ref }}, \widetilde{\mathbf{Q}}^{\text {ref }}$ and $-\widetilde{\mathbf{A}}^{\text {inc }} \widetilde{\mathbf{Q}}^{\text {inc }}$, respectively). This approach has been investigated by several authors, when $\mathbf{A}$ and $\mathbf{b}$ are perturbed as $\mathbf{A}+\Delta \mathbf{A}$ and $\mathbf{b}+\Delta \mathbf{b}$, assuming $\operatorname{rank}(\mathbf{A})=\operatorname{rank}(\mathbf{A}+\Delta \mathbf{A})[28,29,30]$ : for example, a first order expression of the relative error $\|\Delta \mathbf{x}\|_{2} /\|\mathbf{x}\|_{2}$ is brought in ref. [24] in case where $\mathbf{A}$ and $\Delta \mathbf{A}$ are full column rank:

$$
\frac{\|\Delta \mathbf{x}\|_{2}}{\|\mathbf{x}\|_{2}} \leq \epsilon \kappa_{2}(\mathbf{A})\left(1+\frac{\|\mathbf{b}\|_{2}}{\|\mathbf{A}\|_{2}\|\mathbf{x}\|_{2}}+\kappa_{2}(\mathbf{A}) \frac{\|\mathbf{b}-\mathbf{A} \mathbf{x}\|_{2}}{\|\mathbf{A}\|\left\|_{2}\right\| \mathbf{x} \|_{2}}\right)+\mathcal{O}\left(\epsilon^{2}\right)
$$

where assumption is made that $\|\Delta \mathbf{A}\|_{2} /\|\mathbf{A}\|_{2} \leq \epsilon$ and $\|\Delta \mathbf{b}\|_{2} /\|\mathbf{b}\|_{2} \leq \epsilon$, and where $\kappa_{2}(\mathbf{A})=\|\mathbf{A}\|_{2}\left\|\mathbf{A}^{+}\right\|_{2}$ is the condition number of $\mathbf{A}$.

Another possibility to carry out this analysis could consist in formulating Eq. (9) by means of a full square system of the form $\mathbf{A x}=\mathbf{b}$, using a projection scheme for reducing the dimension of the original over-dimensioned problem. In that case, the relative error appears simply bounded as [24]:

$$
\frac{\|\Delta \mathbf{x}\|}{\|\mathbf{x}\|} \leq \frac{2 \epsilon \kappa(\mathbf{A})}{1-\epsilon \kappa(\mathbf{A})}
$$

whatever the consistent norm $\|$.$\| used, where \kappa(\mathbf{A})=\|\mathbf{A}\|\left\|\mathbf{A}^{-1}\right\|$ while it is assumed that $\epsilon \kappa(\mathbf{A})<1$.

Expressing $\left\|\Delta_{j} \widetilde{\mathbf{Q}}^{\text {ref }}\right\| /\left\|\widetilde{\mathbf{Q}}^{\text {ref }}\right\|$ by means of Eq. (33) or Eq. (34) is attractive as quite straightforward indicators $\kappa(\mathbf{A})$ and $\epsilon$ are invoked. Unfortunately, these formulations remain confined within the framework of restrictive assumptions - i.e. $\epsilon<<1$ for Eq. (33) and $\epsilon \kappa(\mathbf{A})<1$ for Eq. (34) - that does not seem to be necessarily verified in the present study as junction resonances are considered. In this case, $\kappa(\mathbf{A})$ is expected to reach large values while the fact of perturbing $\mathbb{D}^{*}$ as $\mathbb{D}^{*}+\Delta_{j} \mathbb{D}^{*}$ can yield non-negligible values of $\epsilon$. Such an issue enforces the 
motivation of using Eq. (32) throughout this paper.

The bound $\left\|\Delta_{j} \widetilde{\mathbb{C}}\right\| /\|\widetilde{\mathbb{C}}\|$ in Eq. (32) can be addressed as follows: from Eq. (21), $\widetilde{\mathbb{C}}=-\left(\widetilde{\mathbf{A}}^{\text {ref }}\right)+\widetilde{\mathbf{A}}^{\text {inc }}$ where $\widetilde{\mathbf{A}}^{\text {ref }}$ and $\widetilde{\mathbf{A}}^{\text {inc }}$ are given by Eq. (10). Without loss of generality, $\|$.$\| can be chosen e.g. as the Frobenius norm \|.\|_{F}[24]$. Hence, the bound writes $\left\|\Delta_{j} \widetilde{\mathbb{C}}\right\|_{F} /\|\widetilde{\mathbb{C}}\|_{F}$ and is readily formulated as:

$\frac{\left\|\Delta_{j} \widetilde{\mathbb{C}}\right\|_{F}}{\|\widetilde{\mathbb{C}}\|_{F}}=\frac{\left\|\left(\widetilde{\mathbf{A}}^{\text {ref }}+\Delta_{j} \widetilde{\mathbf{A}}^{\text {ref }}\right)+\left(\widetilde{\mathbf{A}}^{\text {inc }}+\Delta_{j} \widetilde{\mathbf{A}}^{\text {inc }}\right)-\left(\widetilde{\mathbf{A}}^{\text {ref }}\right)+\widetilde{\mathbf{A}}^{\text {inc }}\right\|_{F}}{\left\|\left(\widetilde{\mathbf{A}}^{\text {ref }}\right)+\widetilde{\mathbf{A}}^{\text {inc }}\right\|_{F}} \quad j=1, \ldots, n_{\mathrm{I}}^{\mathrm{c}}$,

where

$\Delta_{j} \widetilde{\mathbf{A}}^{\text {ref }}=\mathbf{T}^{T} \Delta_{j} \mathbb{D}^{*} \mathbf{T} \widetilde{\boldsymbol{\Psi}}_{\mathrm{q}}^{\mathrm{ref}} \quad, \quad \Delta_{j} \widetilde{\mathbf{A}}^{\text {inc }}=\mathbf{T}^{T} \Delta_{j} \mathbb{D}^{*} \mathbf{T} \widetilde{\boldsymbol{\Psi}}_{\mathrm{q}}^{\text {inc }} \quad j=1, \ldots, n_{\mathrm{I}}^{\mathrm{c}}$.

The error $\left\|\Delta_{j} \widetilde{\mathbb{C}}\right\|_{F} /\|\widetilde{\mathbb{C}}\|_{F}$ has to be computed for every discrete frequency $\omega_{k} / 2 \pi$ $\left(k=1, \ldots, N_{f}\right)$ considered within the frequency band $\mathcal{B}_{f}$. One straightforward way to address this issue consists in assessing $\left\|\Delta_{j} \widetilde{\mathbb{C}}\right\|_{F} /\|\widetilde{\mathbb{C}}\|_{F}$ as its maximum value within $\mathcal{B}_{f}$. This yields:

$$
\frac{\left\|\Delta_{j} \widetilde{\mathbf{Q}}_{\omega_{p}}^{\mathrm{ref}}\right\|_{F}}{\left\|\widetilde{\mathbf{Q}}_{\omega_{p}}^{\mathrm{ref}}\right\|_{F}} \leq \max \left\{\frac{\left\|\Delta_{j} \widetilde{\mathbb{C}}_{\omega_{k}}\right\|_{F}}{\left\|\widetilde{\mathbb{C}}_{\omega_{k}}\right\|_{F}}\right\}_{k=1, \ldots, N_{f}} \quad \forall \omega_{p} \quad j=1, \ldots, n_{\mathrm{I}}^{\mathrm{c}},
$$

where the subscripts $\omega_{p}$ and $\omega_{k}$ refer to as the pulsations used for calculating the vector / matrix terms. Eq. (37) addresses the impact of neglecting one junction mode $j$ when computing the forced response of the coupled system. It is therefore proposed to retain the junction modes for which the bound in Eq. (37) is greater than a specified threshold, and to neglect the others. Hereafter, the selection criterion (37) is compared to the usual strategy that consists in sorting the modes in ascending order. Its relevance is also discussed. 


\subsection{Numerical results}

The wave-based matrix formulation (23) is applied for computing the forced response of the coupled system depicted in Figure 1 and whose characteristics have been detailed in subsection 2.4. In the CMS framework, the condensed form $\widetilde{\mathbb{D}}^{*}$ of the junction dynamic stiffness matrix is constructed by means of a reduced family of elastic modes (cf. Eq. (29)). The strategy used for selecting these junction modes constitutes the key point of the following study; the issue is to test the convergence of the wave-based matrix formulation (23) for capturing the dynamics of the coupled system over the frequency band of interest, i.e. $\mathcal{B}_{f}=[10 \mathrm{~Hz}, 5000$ $\mathrm{Hz}]$ in the present case (cf. subsection 2.4).

One first attempt to select these modes is to use the conventional "ascending order" strategy, consisting in retaining the modes whose eigenfrequencies are below a certain frequency limit while rejecting the others. The procedure for defining this limit accurately is not a simple task: this indeed depends on the degree of complexity, a priori unknown, required to capture the junction spatial dynamics and coupling conditions. This explains why this procedure is rather empirical, i.e. using an arbitrary number of junction modes (sorted in ascending order) as a test case and analyzing the convergence of the formulation in a post-processing step. In this framework, it is thus proposed to construct $\widetilde{\mathbb{D}}^{*}$ by means of arbitrary numbers of junction modes, e.g. $m^{\mathrm{c}}=0^{12}, m^{\mathrm{c}}=10, m^{\mathrm{c}}=20$ and $m^{\mathrm{c}}=30$. The first 30 junction eigenfrequencies are presented in Figure 9, where the maximum value of $\mathcal{B}_{f}$ - i.e. $5000 \mathrm{~Hz}$ - is depicted by a violet line.

\section{Figure 9}

\footnotetext{
${ }^{12}$ In that case, the response of the junction is rather static as it is driven by the constraint modes $\left\{\left(\widetilde{\mathbf{X}}_{\mathrm{st}}\right)_{j}\right\}_{j}$ only.
} 
Using these different numbers $m^{c}$ of junction modes for constructing $\widetilde{\mathbb{D}}^{*}$ and computing the solutions of the wave-based matrix formulation (23) yields the forced response of the coupled system. To this end, the behavior of each waveguide is assessed by means of $m=50$ wave modes (indeed, the relevance of such a wave mode expansion has been established in subsection 2.4). The results are shown in Figure 10.

Expressing the static response of the junction - i.e. using $m^{c}=0$ elastic modes - clearly yields a lack of accuracy of the WFE formulation above $2500 \mathrm{~Hz}$, i.e. when the junction exhibits local dynamics. Enlarging the number of junction modes used in Eq. (23) solves this issue, as expected. When $m^{c}=20$, slight differences between WFE and reference FE solutions still persist above $4000 \mathrm{~Hz}$, even though the junction resonances are completely covered over $\mathcal{B}_{f}$ by the reduced basis $\left\{\left(\widetilde{\mathbf{X}}_{\mathrm{el}}\right)_{j}\right\}_{j}$ (the highest retained eigenfrequency is actually up to 6000 $\mathrm{Hz}$ ). The issue is that the family of junction modes is not rich enough for capturing the wave reflection / transmission phenomena across the junction. Accounting for ten additional higher order modes (with additional eigenfrequencies between 6000 $\mathrm{Hz}$ and $8000 \mathrm{~Hz}$ ) finally yields the convergence of the wave-based formulation.

\section{Figure 10}

To summarize, the ascending order strategy consists in selecting an arbitrary number of junction modes, then computing the WFE solution by means of Eq. (23) and analyzing the convergence of the wave-based matrix formulation in a post-processing step. When the convergence is not reached, the number of modes $m^{\mathrm{c}}$ is increased and the convergence tested again. Apart from this repetitive postprocessing procedure, the major drawback of this strategy is that it could yield an excessive number of elastic modes $\left\{\left(\widetilde{\mathbf{X}}_{\mathrm{el}}\right)_{j}\right\}_{j}$ to be accounted for, part of them being of weak influence (whatever their eigenfrequencies). 
Otherwise, using Eq. (37) leads to the selection of junction modes that effectively contribute to the system forced response. Eq. (37) yields a bound of the relative error $\left\|\Delta_{j} \widetilde{\mathbf{Q}}^{\text {ref }}\right\| /\left\|\widetilde{\mathbf{Q}}^{\text {ref }}\right\|$ that affects the WFE method when neglecting one mode $j$. A small bound means that a mode $j$ can be rejected away when computing the solution of Eq. (23), with small impact. The bounds associated with the first 30 junction modes are depicted in Figure 11.

\section{Figure 11}

The magnitudes of these bounds do not appear necessarily linked to the ranks of the modes, contrary to what is implicitly considered with the ascending order strategy. Using Eq. (37) constitutes an efficient means of selecting the contributing modes, considering bounds that exceed for instance a threshold of $10 \%$. In that case, this yields 19 junction modes - i.e. modes 1 to 17,19 and $22-$ to be selected among the former 30. The WFE solution obtained using these 19 elastic modes is plotted in Figure 12; it appears coherent with the reference FE solution and comparable with the solutions derived from the ascending order strategy with 30 modes (see Figure 10). Precisely, the convergence of both strategies can be underlined when analyzing the relative error of the forced response (at the measurement point) over the whole frequency band $\mathcal{B}_{f}{ }^{13}$. For this task, it is proposed to deal with the quadratic acceleration $\Gamma_{\text {mes }}^{2}=\left(\omega^{2}\left\|\mathbf{q}_{\text {mes }}\right\|_{2}\right)^{2}$ instead of $\left\|\mathbf{q}_{\text {mes }}\right\|_{2}$ ( $\mathbf{q}_{\text {mes }}$ being the displacement vector at the measurement point) "to strenghten" the influence of junction modes with high eigenfrequencies. A 2-norm is introduced to address the relative error over the frequency band $\mathcal{B}_{f}$, the latter being expressed

\footnotetext{
${ }^{13}$ Such an approach has been discussed in ref. [31] with regard to the transfer functions of linear systems.
} 
in terms of dicrete frequencies $\left\{\omega_{k} / 2 \pi\right\}_{k=1, \ldots, N_{f}}$. This norm is defined as:

$$
\left\|\Gamma_{m e s}^{2}\right\|_{\mathcal{B}_{f}}=\left(\sum_{k=1}^{N_{f}}\left(\Gamma_{m e s}^{2}\right)_{\omega_{k}}^{2}\right)^{\frac{1}{2}} .
$$

where the subscript $\omega_{k}$ refers to as the pulsation used for calculating the quadratic acceleration. The relative error thus writes as:

$$
\mathcal{E}^{\mathrm{WFE}}=\frac{\left\|\left(\Gamma_{m e s}^{2}\right)^{\mathrm{WFE}}-\left(\Gamma_{m e s}^{2}\right)^{\mathrm{FE}}\right\|_{\mathcal{B}_{f}}}{\left\|\left(\Gamma_{m e s}^{2}\right)^{\mathrm{FE}}\right\|_{\mathcal{B}_{f}}}
$$

where $\left(\Gamma_{m e s}^{2}\right)^{\mathrm{WFE}}$ and $\left(\Gamma_{m e s}^{2}\right)^{\mathrm{FE}}$ represent the WFE solution and the reference solution provided by FE, respectively. The relative errors for both strategies depicted above are plotted in Figure 13 as functions of the number of junction modes retained in the WFE formulation. As expected, the strategy based on Eq. (37) yields the convergence to be reached efficiently with a few junction modes. The fact that junction modes are ranked in accordance to their contribution enables the error to decrease uniformly compared to the conventional ascending order strategy. It appears that retaining 19 modes when using criterion (37) provides the same relative error as the conventional strategy with 22 modes. Of course this last result (i.e. 22 modes for the conventional strategy) has been established since the system forced response has been computed several times (i.e. considering wave bases of different dimensions as test cases for computing the error). The selection strategy based on criterion (37) circumvents such an issue by investigating the contribution of each mode in a pre-processing step. To summarize, the feature of Eq. (37) is that the number of junction modes retained in the wave-based formulation can be reduced compared to the conventional procedure; the norm-wise strategy also circumvents the way to proceed empirically for testing the convergence of the formulation in several time consuming post-processing steps.

\section{Figure 12}




\section{Perturbation analysis}

\subsection{Introduction}

A CMS-based WFE approach has been investigated in the previous subsection for computing the forced response of coupled systems involving elastic junctions. Emphasis was on a model order reduction strategy using a few number of junction modes. This yields the CPU time to be reduced further which is attractive when analyzing for instance slight uncertainties of junction modes by means of Monte Carlo simulations (MCS). A perturbation analysis that addresses those uncertainties is proposed in this section. Again, the case of two waveguides connected with one junction is investigated. In particular, forward component-wise bounds of the WFE solution $\mathbf{u}_{i}$ (i.e. the displacements and internal forces within each waveguide $i$ ) are derived. The underlying assumptions of this perturbation analysis are as follows:

- The eigenpulsation $\widetilde{\omega}_{j}$ of each junction mode $j$ is perturbed as $\widetilde{\omega}_{j}^{0}+\delta \widetilde{\omega}_{j}$, where:

$$
\left|\delta \widetilde{\omega}_{j} / \widetilde{\omega}_{j}^{0}\right| \leq \theta_{j}<<1 \quad j=1, \ldots, m^{\mathrm{c}} .
$$

Here, $\widetilde{\omega}_{j}^{0}$ refers to as the baseline eigenpulsation, while $\theta_{j}$ represents a deterministic bound of $\left|\delta \widetilde{\omega}_{j} / \widetilde{\omega}_{j}^{0}\right|$;

- The constraint modes $\left\{\left(\widetilde{\mathbf{X}}_{\mathbf{s t}}\right)_{j}\right\}_{j}$ and elastic modes $\left\{\left(\widetilde{\mathbf{X}}_{\mathrm{el}}\right)_{j}\right\}_{j}$ are unperturbed ${ }^{14}$;

- For each waveguide $i$, the wave modes $\left\{\left(\left(\widetilde{\mu}_{j}\right)_{i},\left(\widetilde{\boldsymbol{\Phi}}_{j}\right)_{i}\right)\right\}_{j}$ are unperturbed.

\footnotetext{
${ }^{14}$ Such an assumption is commonly used in the literature [16].
} 
The issue to provide forward component-wise bounds of the perturbed vector $\mathbf{u}_{i}=$ $\mathbf{u}^{0}+\delta \mathbf{u}_{i}$, using first order Taylor series expansions ${ }^{15}$, is the key idea of the present study. Comparisons with the results of MCS are investigated in subsection 4.3.

\subsection{Bounds of $\mathbf{u}_{i}$}

For each waveguide $i$, the state vector $\mathbf{u}_{i}$ is expressed as in Eq. (2) - say, in terms of displacement vector $\mathbf{q}_{i}$ and force vector $\mathbf{F}_{i}$ - for a cross-section located at longitudinal position $k_{i} d_{i}\left(k_{i}=1, \ldots, N_{i}+1 ; d_{i}\right.$ being the length of a typical substructure). According to the wave mode expansion $\mathbf{u}_{i} \approx \widetilde{\boldsymbol{\Phi}}_{i} \widetilde{\mathbf{Q}}_{i}$ and since $\widetilde{\boldsymbol{\Phi}}_{i}$ is considered as unperturbed, $\mathbf{u}_{i}=\mathbf{u}_{i}^{0}+\delta \mathbf{u}_{i}$ can be assessed as $\widetilde{\boldsymbol{\Phi}}_{i}\left(\widetilde{\mathbf{Q}}_{i}^{0}+\delta \widetilde{\mathbf{Q}}_{i}\right)$, i.e. by means of $\delta \widetilde{\mathbf{Q}}_{i}$ only. The related component-wise bounds are investigated hereafter:

According to Eq. (5), the wave mode amplitudes $\widetilde{\mathbf{Q}}_{i}$ at position $k_{i} d_{i}$ are linked to those $\widetilde{\mathbf{Q}}_{i}^{(1)}$ expressed at one of the waveguide boundaries (e.g. where excitations are imposed). This writes as:

$$
\widetilde{\mathbf{Q}}_{i}=\widetilde{\mathbf{Q}}_{i}^{\left(k_{i}\right)}=\widetilde{\mathcal{M}}_{i} \widetilde{\mathbf{Q}}_{i}^{(1)} \quad k_{i}=1, \ldots, N_{i}+1, \quad i=1,2,
$$

where

$$
\widetilde{\mathcal{M}}_{i}=\left[\begin{array}{cc}
\widetilde{\boldsymbol{\mu}}_{i}^{k_{i}-1} & \mathbf{0} \\
\mathbf{0} & \widetilde{\boldsymbol{\mu}}_{i}^{-\left(k_{i}-1\right)}
\end{array}\right] \quad k_{i}=1, \ldots, N_{i}+1, \quad i=1,2 .
$$

This yields a simple way to express $\delta \widetilde{\mathbf{Q}}_{i}$ by means of $\delta \widetilde{\mathbf{Q}}_{i}^{(1)}$, as $\widetilde{\mathcal{M}}_{i}$ remains unperturbed (cf. subsection 4.1). The perturbed vector $\delta \widetilde{\mathbf{Q}}_{i}^{(1)}$ can be assessed by means of Eq. (23); in abridged notations, this writes:

$$
\widetilde{\mathcal{A}} \widetilde{\mathbb{C}} \widetilde{\mathcal{Q}}=\widetilde{\mathcal{F}}
$$

\footnotetext{
${ }^{15}$ In this framework, the superscript 0 denotes the solutions computed when $\widetilde{\omega}_{j}=\widetilde{\omega}_{j}^{0} \forall j$.
} 
where $\widetilde{\mathcal{Q}}=\left[\widetilde{\mathbf{Q}}_{1}^{(1) T} \widetilde{\mathbf{Q}}_{2}^{(1) T}\right]^{T} ; \widetilde{\mathcal{A}}_{\widetilde{\mathbb{C}}}$ represents the first matrix in Eq. (23) which is square and linked to the components of $\widetilde{\mathbb{C}}$, the latter being constructed by means of the junction modes (cf. previous subsection); the remaining terms $\widetilde{\mathcal{D}}$ and $\widetilde{\mathcal{F}}$ do not depend on the junction modes: $\widetilde{\mathcal{D}}$ represents the second matrix in Eq. (23) which is diagonal, while $\widetilde{\mathcal{F}}$ reflects the excitation sources. According to this, taking into account that $\widetilde{\mathcal{Q}}$ is formulated as $\widetilde{\mathcal{Q}}=\widetilde{\mathcal{D}}^{-1} \widetilde{\mathcal{A}}_{\widetilde{\mathbb{C}}}^{-1} \widetilde{\mathcal{F}}$ (cf. Eq. (43)) yields the derivative $\partial \widetilde{\mathcal{Q}} / \partial \widetilde{\omega}_{j}$ to be written as

$$
\frac{\partial \widetilde{\mathcal{Q}}}{\partial \widetilde{\omega}_{j}}=\widetilde{\mathcal{D}}^{-1}\left(-\widetilde{\mathcal{A}}_{\widetilde{\mathbb{C}}}^{-1} \frac{\partial \widetilde{\mathcal{A}}_{\widetilde{\mathbb{C}}}}{\partial \widetilde{\omega}_{j}} \widetilde{\mathcal{A}}_{\widetilde{\mathbb{C}}}^{-1}\right) \widetilde{\mathcal{F}} \quad j=1, \ldots, m^{c},
$$

where the term between the brackets denotes the derivative of $\mathcal{A}_{\mathbb{C}}^{-1}$; according to Eq. (23), the derivative $\partial \mathcal{A}_{\mathbb{C}} / \partial \widetilde{\omega}_{j}$ in Eq. (44) readily writes by means of the derivative $\partial \widetilde{\mathbb{C}} / \partial \widetilde{\omega}_{j}$ (cf. Appendix B) as:

$$
\frac{\partial \widetilde{\mathcal{A}}_{\widetilde{\mathbb{C}}}}{\partial \widetilde{\omega}_{j}}=\left[\begin{array}{cc|cc}
\mathbf{0} & \mathbf{0} & \mathbf{0} & \mathbf{0} \\
-\frac{\partial \widetilde{\mathbb{C}}_{11}}{\partial \widetilde{\omega}_{j}} \widetilde{\boldsymbol{\mu}}_{1}^{N_{1}} & \mathbf{0} & \mathbf{0} & -\frac{\partial \widetilde{\mathbb{C}}_{12}}{\partial \widetilde{\omega}_{j}} \widetilde{\boldsymbol{\mu}}_{2}^{N_{2}} \\
\hline \partial \widetilde{\mathbb{C}}_{21} \widetilde{\boldsymbol{\mu}}_{1}^{N_{1}} & \mathbf{0} & \mathbf{0} & -\frac{\partial \widetilde{\mathbb{C}}_{22}}{\partial \widetilde{\omega}_{j}} \widetilde{\boldsymbol{\mu}}_{2}^{N_{2}} \\
\mathbf{0} & \mathbf{0} & \mathbf{0} & \mathbf{0}
\end{array}\right] \quad j=1, \ldots, m^{\mathrm{c}}
$$

As a result, using first order Taylor series expansion yields the variation $\delta \widetilde{\mathcal{Q}}$ as:

$$
\delta \widetilde{\mathcal{Q}}=\sum_{j=1}^{m^{c}}\left(\frac{\partial \widetilde{\mathcal{Q}}}{\partial \widetilde{\omega}_{j}}\right)^{0} \delta \widetilde{\omega}_{j},
$$

where $\left(\partial \widetilde{\mathcal{Q}} / \partial \widetilde{\omega}_{j}\right)^{0}$ follows from Eqs. (44) and (45) when $\widetilde{\omega}_{j}=\widetilde{\omega}_{j}^{0}$. The derivation of $\delta \widetilde{\mathbf{Q}}_{i}^{(1)}$ results from Eq. (46), while the formulation of component-wise bounds of $\delta \mathbf{u}_{i}$ follows from the wave mode expansion procedure summarized at the beginning of this subsection:

$$
\left|\delta \mathbf{u}_{i}\right| \leq \sum_{j=1}^{m^{c}}\left|\widetilde{\boldsymbol{\Phi}}_{i} \widetilde{\mathcal{M}}_{i}\left(\frac{\partial \widetilde{\mathbf{Q}}_{i}^{(1)}}{\partial \widetilde{\omega}_{j}}\right)^{0}\right| \widetilde{\omega}_{j}^{0} \theta_{j} \quad i=1,2,
$$


where |.| denotes the operation of replacing each element of a matrix by its magnitude or its absolute value (i.e. should the real or imaginary part of $\mathbf{u}_{i}$ be required). Thus, the bounds of $\mathbf{u}_{i}$ can be expressed as:

- Case when the magnitude of $\mathbf{u}_{i}$ is required:

$$
\left|\mathbf{u}_{i}^{0}\right|-\sum_{j=1}^{m^{c}}\left|\widetilde{\mathbf{\Phi}}_{i} \widetilde{\mathcal{M}}_{i}\left(\frac{\partial \widetilde{\mathbf{Q}}_{i}}{\partial \widetilde{\omega}_{j}}\right)^{0}\right| \widetilde{\omega}_{j}^{0} \theta_{j} \leq\left|\mathbf{u}_{i}\right| \leq\left|\mathbf{u}_{i}^{0}\right|+\sum_{j=1}^{m^{c}}\left|\widetilde{\mathbf{\Phi}}_{i} \widetilde{\mathcal{M}}_{i}\left(\frac{\partial \widetilde{\mathbf{Q}}_{i}}{\partial \widetilde{\omega}_{j}}\right)^{0}\right| \widetilde{\omega}_{j}^{0} \theta_{j} \quad i=1,2 ;
$$

- Case when the real or imaginary part of $\mathbf{u}_{i}$ is required:

$$
\mathbf{u}_{i}^{0}-\sum_{j=1}^{m^{c}}\left|\widetilde{\mathbf{\Phi}}_{i} \widetilde{\mathcal{M}}_{i}\left(\frac{\partial \widetilde{\mathbf{Q}}_{i}}{\partial \widetilde{\omega}_{j}}\right)^{0}\right| \widetilde{\omega}_{j}^{0} \theta_{j} \leq \mathbf{u}_{i} \leq \mathbf{u}_{i}^{0}+\sum_{j=1}^{m^{c}}\left|\widetilde{\mathbf{\Phi}}_{i} \widetilde{\mathcal{M}}_{i}\left(\frac{\partial \widetilde{\mathbf{Q}}_{i}}{\partial \widetilde{\omega}_{j}}\right)^{0}\right| \widetilde{\omega}_{j}^{0} \theta_{j} \quad i=1,2,
$$

where $\mathbf{u}_{i}$ and $|$.$| have to be understood as \operatorname{Re}\left(\mathbf{u}_{i}\right)$ and $|\operatorname{Re}()$.$| , or \operatorname{Im}\left(\mathbf{u}_{i}\right)$ and $|\operatorname{Im}()$.$| .$

\subsection{Numerical results}

Component-wise bounds of the state vectors $\left\{\mathbf{u}_{i}\right\}_{i=1,2}$ are investigated over the frequency band $\mathcal{B}_{f}=[10 \mathrm{~Hz}, 5000 \mathrm{~Hz}]$. The test case depicted in Figure 1 is considered. The wave-based numerical formulation (23) is addressed when the coupling junction model uses $m^{c}=19$ elastic modes with uncertain eigenpulsations $\left\{\widetilde{\omega}_{j}^{0}+\delta \widetilde{\omega}_{j}\right\}_{j}$. These junction modes are selected by means of the criterion (37) discussed in subsection 3.4. It is assumed that $\left|\delta \widetilde{\omega}_{j} / \widetilde{\omega}_{j}^{0}\right| \leq \theta_{j}=5 \% \forall j$, where $\left\{\widetilde{\omega}_{j}^{0}\right\}_{j}$ denote the baseline eigenpulsations (cf. Figure 9). The componentwise bounds of the state vectors $\left\{\mathbf{u}_{i}\right\}_{i=1,2}$ are addressed by means of Eq. (48). The state vectors provide the displacements and internal forces within waveguides 
1 and 2 (see Section 2). Particularly, the displacement of waveguide 1 is investigated at the measurement point, where forces are imposed. The baseline solution represents the forced response computed using the CMS-based WFE formulation with $m=50$ wave modes and $m^{\mathrm{c}}=19$ junction modes (cf. above). The bounds of the transverse displacement are depicted in Figure 14. As a logarithmic scale is used, the higher bound is of primary importance since the lower bound may be badly interpreted as involving close to zero or negative values ${ }^{16}$. As expected, the bounds appear of primary importance when the junction exhibits local dynamics, i.e. above $2500 \mathrm{~Hz}$. They exhibit large values around the local extrema of the baseline solution (cf. Figure 14(a)), when the junction resonances are reached. Monte Carlo simulations (MCS) are carried out to test the relevance of the bounds (cf. Figure 14(b)). To this end, 100 sets of random values $\left\{\widetilde{\omega}_{j}^{0}+\delta \widetilde{\omega}_{j}\right\}_{j}$ are used as trials, each $\delta \widetilde{\omega}_{j}$ following a uniform distribution over the range $\left|\delta \widetilde{\omega}_{j} / \widetilde{\omega}_{j}^{0}\right| \leq \theta_{j}$. For each trial, the components of $\left\{\mathbf{u}_{i}\right\}_{i=1,2}$ are computed by means of Eq. (23). It is worth emphasizing that solving the small WFE matrix system (23) leads to significant CPU time savings compared to the usual FE approach (i.e. in case where the full FE model of waveguides could be considered), say several seconds against several minutes for each trial. As expected, the bounds provided by Eq. (48) appear valid when compared to the results of MCS, in the sense they provide reasonable confidence areas.

\section{Figure 14}

\footnotetext{
${ }^{16}$ One artefact to assess the logarithm of this bound is to remove negative values and to consider positive close-to zero terms instead.
} 


\section{Concluding remarks}

The low- and mid-frequency forced response of coupled systems involving straight structures connected with elastic junctions has been addressed using the wave finite element (WFE) method. In this framework, the kinematic fields of each straight structure are expanded in terms of numerical wave modes having a one-dimensional propagation, while the junction dynamics are captured using classic FE procedures. One feature of this study is that it uses a Lagrange Multipliers formalism so as to relax mesh compatibility assumption over coupling junction interfaces. The resulting mesh tying formulation provides an efficient means for computing the magnitudes of reflected waves from those that are incident towards any given junction. The relevance of this formalism has been discussed from a computational point of view; it has been highlighted for computing the forced response of two beam-like structures coupled transversally through a quarter of torus that undergoes local resonances. Also, a CMS-based WFE formulation that uses junction elastic modes has been investigated. A strategy has been proposed for reducing efficiently the number of modes retained in the formulation. In this framework, a norm-wise criterion has been derived for selecting those junction modes that are of primary importance for computing the system forced response. Finally, a perturbation analysis has been proposed for assessing the system forced response when slight uncertainties affect the junction eigenfrequencies. Component-wise perturbation bounds have been formulated for the WFE solutions. The relevance of the model has been emphasized through comparisons with Monte Carlo simulations. 


\section{Appendix A. Derivation of power reflection / transmission coefficients}

Let $P_{i}^{\mathrm{inc}}$ and $P_{i}^{\text {ref }}$ be the energy flows traveling in waveguide $i$ towards and outwards the junction, respectively. These can be defined as $P_{i}^{\text {inc }}=(\omega / 2) \operatorname{Im}\left(\left(\mathbf{q}_{\mathrm{L}}^{\text {inc }}\right)_{i}^{H}\left(\mathbf{F}_{\mathrm{L}}^{\text {inc }}\right)_{i}\right)$ and $P_{i}^{\text {ref }}=-(\omega / 2) \operatorname{Im}\left(\left(\mathbf{q}_{\mathrm{L}}^{\text {ref }}\right)_{i}^{H}\left(\mathbf{F}_{\mathrm{L}}^{\text {ref }}\right)_{i}\right)$, that is [32]:

$$
P_{i}^{\text {inc }}=\frac{i \omega}{4}\left(\mathbf{u}_{i}^{\text {inc }}\right)^{H} \mathbf{J} \mathbf{u}_{i}^{\text {inc }} \quad, \quad P_{i}^{\text {ref }}=-\frac{i \omega}{4}\left(\mathbf{u}_{i}^{\text {ref }}\right)^{H} \mathbf{J} \mathbf{u}_{i}^{\text {ref }} \quad \forall i, \quad(\mathrm{~A}-1)
$$

where $\mathbf{u}_{i}^{\text {inc }}=\sum_{j}\left(Q_{j}^{\text {inc }}\right)_{i}\left(\Phi_{j}^{\text {inc }}\right)_{i}$ and $\mathbf{u}_{i}^{\text {ref }}=\sum_{j}\left(Q_{j}^{\text {ref }}\right)_{i}\left(\boldsymbol{\Phi}_{j}^{\text {ref }}\right)_{i}$, while $\mathbf{J}_{i}$ is given as

$$
\mathbf{J}_{i}=\left[\begin{array}{rc}
\mathbf{0} & \mathbf{I}_{n_{i}} \\
-\mathbf{I}_{n_{i}} & \mathbf{0}
\end{array}\right] \quad \forall i .
$$

It is commonly stated that energy flows resulting from wave interferences can be neglected [25]. As a result, this yields

$$
P_{i}^{\mathrm{inc}}=\sum_{j}\left(P_{j}^{\mathrm{inc}}\right)_{i} \quad, \quad P_{i}^{\mathrm{ref}}=\sum_{j}\left(P_{j}^{\mathrm{ref}}\right)_{i} \quad \forall i
$$

where

$$
\begin{aligned}
& \left(P_{j}^{\text {inc }}\right)_{i}=\frac{i \omega}{4}\left(\boldsymbol{\Phi}_{j}^{\text {inc }}\right)_{i}^{H} \mathbf{J}_{i}\left(\boldsymbol{\Phi}_{j}^{\text {inc }}\right)_{i}\left|\left(Q_{j}^{\text {inc }}\right)_{i}\right|^{2} \quad \forall j, \\
& \left(P_{j}^{\text {ref }}\right)_{i}=-\frac{i \omega}{4}\left(\boldsymbol{\Phi}_{j}^{\text {ref }}\right)_{i}^{H} \mathbf{J}_{i}\left(\boldsymbol{\Phi}_{j}^{\text {ref }}\right)_{i}\left|\left(Q_{j}^{\text {ref }}\right)_{i}\right|^{2} \quad \forall j .
\end{aligned}
$$

Also note that $\left(Q_{j}^{\text {ref }}\right)_{i}=\sum_{r} \sum_{s}\left(\mathbb{C}_{i r}\right)_{j s}\left(Q_{s}^{\text {inc }}\right)_{r}$, where $\left(\mathbb{C}_{i r}\right)_{j s}$ refers to as the component $j s$ of block matrix $\mathbb{C}_{i r}$ (cf. Eq. (22)). Neglecting wave interferences, it turns out that each energy flow $\left(P_{j}^{\text {ref }}\right)_{i}$ is expressed as:

$$
\left(P_{j}^{\mathrm{ref}}\right)_{i}=-\frac{i \omega}{4}\left(\boldsymbol{\Phi}_{j}^{\mathrm{ref}}\right)_{i}^{H} \mathbf{J}_{i}\left(\boldsymbol{\Phi}_{j}^{\mathrm{ref}}\right)_{i} \sum_{r} \sum_{s}\left|\left(\mathbb{C}_{i r}\right)_{j s}\right|^{2}\left|\left(Q_{s}^{\text {inc }}\right)_{r}\right|^{2} \quad \forall j . \text { (A-6) }
$$

Let us denote as $\left(P_{j}^{\text {ref }}\right)_{i}^{r s}$ the term $-(i \omega / 4)\left(\boldsymbol{\Phi}_{j}^{\text {ref }}\right)_{i}^{H} \mathbf{J}_{i}\left(\boldsymbol{\Phi}_{j}^{\text {ref }}\right)_{i}\left|\left(\mathbb{C}_{i r}\right)_{j s}\right|^{2}\left|\left(Q_{s}^{\text {inc }}\right)_{r}\right|^{2}$. Thus the reflection and transmission coefficients for an incident energy flow $\left(P_{s}^{\text {inc }}\right)_{r}$ 
traveling along any waveguide $r$ readily write as (respectively):

$$
\begin{array}{cc}
\tau_{r j}^{r s}=\frac{\left(P_{j}^{\text {ref }}\right)_{r}^{r s}}{\left(P_{s}^{\text {inc }}\right)_{r}}=-\frac{\left(\boldsymbol{\Phi}_{j}^{\text {ref }}\right)_{r}^{H} \mathbf{J}_{r}\left(\boldsymbol{\Phi}_{j}^{\text {ref }}\right)_{r}}{\left(\boldsymbol{\Phi}_{s}^{\text {inc }}\right)_{r}^{H} \mathbf{J}_{r}\left(\boldsymbol{\Phi}_{s}^{\text {inc }}\right)_{r}}\left|\left(\mathbb{C}_{r r}\right)_{j s}\right|^{2} & r=i, \\
\tau_{i j}^{r s}=\frac{\left(P_{j}^{\text {ref }}\right)_{i}^{r s}}{\left(P_{s}^{\text {inc }}\right)_{r}}=-\frac{\left(\boldsymbol{\Phi}_{j}^{\text {ref }}\right)_{i}^{H} \mathbf{J}_{i}\left(\boldsymbol{\Phi}_{j}^{\text {ref }}\right)_{i}}{\left(\boldsymbol{\Phi}_{s}^{\text {inc }}\right)_{r}^{H} \mathbf{J}_{r}\left(\boldsymbol{\Phi}_{s}^{\text {inc }}\right)_{r}}\left|\left(\mathbb{C}_{i r}\right)_{j s}\right|^{2} & r \neq i .
\end{array}
$$

\section{Appendix B. Derivation of $\partial \widetilde{\mathbb{C}} / \partial \widetilde{\omega}_{j}$}

The matrix $\widetilde{\mathbb{C}}$ is formulated as $-\left(\widetilde{\mathbf{A}}^{\text {ref }}\right)+\widetilde{\mathbf{A}}^{\text {inc }}$ (cf. Eq. (21)) where, in the CMS framework, $\widetilde{\mathbf{A}}^{\text {ref }}$ and $\widetilde{\mathbf{A}}^{\text {inc }}$ are expressed as

$$
\widetilde{\mathbf{A}}^{\text {ref }}=\mathbf{T}^{T} \widetilde{\mathbb{D}}^{*} \mathbf{T} \widetilde{\mathbf{\Psi}}_{\mathrm{q}}^{\mathrm{ref}}+\widetilde{\mathbf{\Psi}}_{\mathrm{F}}^{\mathrm{ref}} \quad, \quad \widetilde{\mathbf{A}}^{\text {inc }}=\mathbf{T}^{T} \widetilde{\mathbb{D}}^{*} \mathbf{T} \widetilde{\boldsymbol{\Psi}}_{\mathrm{q}}^{\text {inc }}+\widetilde{\mathbf{\Psi}}_{\mathrm{F}}^{\text {inc }}
$$

In that case, $\widetilde{\mathbb{D}}^{*}$ refers to as the junction dynamic stiffness matrix approximated by means of Eq. (29) when using $m^{\mathrm{c}}$ relevant junction modes (i.e. selected by means of criterion (37)). The derivative of $\widetilde{\mathbb{C}}$ with respect to $\widetilde{\omega}_{j}$ readily writes as

$$
\begin{aligned}
\frac{\partial \widetilde{\mathbb{C}}}{\partial \widetilde{\omega}_{j}}= & -\left(\widetilde{\mathbf{A}}^{\text {ref }}\right)^{+}\left[\left(-\frac{\partial \widetilde{\mathbf{A}}^{\text {ref }}}{\partial \widetilde{\omega}_{j}}\left(\widetilde{\mathbf{A}}^{\text {ref }}\right)^{+}+\left(\widetilde{\mathbf{A}}^{\text {ref }}\right)^{+H}\right.\right. \\
& \left.\left.\times\left(\frac{\partial \widetilde{\mathbf{A}}^{\text {ref }}}{\partial \widetilde{\omega}_{j}}\right)^{H}\left(\mathbf{I}-\widetilde{\mathbf{A}}^{\text {ref }}\left(\widetilde{\mathbf{A}}^{\text {ref }}\right)^{+}\right)\right) \widetilde{\mathbf{A}}^{\text {inc }}+\frac{\partial \widetilde{\mathbf{A}}^{\text {inc }}}{\partial \widetilde{\omega}_{j}}\right] \quad j=1, \ldots, m^{\mathrm{c}},
\end{aligned}
$$

where the matrix term $\left(\mathbf{I}-\widetilde{\mathbf{A}}^{\text {ref }}\left(\widetilde{\mathbf{A}}^{\text {ref }}\right)^{+}\right)$is linked to the derivative of the pseudoinverse $\left(\widetilde{\mathbf{A}}^{\text {ref }}\right)^{+}[28]^{17}$. In Eq. (B-2), the derivatives $\partial \widetilde{\mathbf{A}}^{\text {ref }} / \partial \widetilde{\omega}_{j}$ and $\partial \widetilde{\mathbf{A}}^{\text {inc }} / \partial \widetilde{\omega}_{j}$ are formulated by means of Eq. (B-1) as

$$
\frac{\partial \widetilde{\mathbf{A}}^{\text {ref }}}{\partial \widetilde{\omega}_{j}}=\mathbf{T}^{T} \frac{\partial \widetilde{\mathbb{D}}^{*}}{\partial \widetilde{\omega}_{j}} \mathbf{T} \widetilde{\mathbf{\Psi}}_{\mathrm{q}}^{\mathrm{ref}} \quad, \quad \frac{\partial \widetilde{\mathbf{A}}^{\text {inc }}}{\partial \widetilde{\omega}_{j}}=\mathbf{T}^{T} \frac{\partial \widetilde{\mathbb{D}}^{*}}{\partial \widetilde{\omega}_{j}} \mathbf{T} \widetilde{\mathbf{\Psi}}_{\mathrm{q}}^{\text {inc }} \quad j=1, \ldots, m^{\mathrm{c}}
$$

\footnotetext{
${ }^{17}$ Notice that the derivative of the classic inverse $\left(\widetilde{\mathbf{A}}^{\text {ref }}\right)^{-1}$ does not invoked such a term.
} 
(B-3)

Here, $\partial \widetilde{\mathbb{D}}^{*} / \partial \widetilde{\omega}_{j}$ is readily expressed by means of Eqs. (29) and (27) as:

$$
\frac{\partial \widetilde{\mathbb{D}}^{*}}{\partial \widetilde{\omega}_{j}}=\widetilde{\mathbb{D}}_{\mathrm{e} 1-\mathrm{st}}^{T} \widetilde{\mathbb{D}}_{\mathrm{el}-\mathrm{el}}^{-1} \frac{\partial \widetilde{\mathbb{D}}_{\mathrm{e} 1-\mathrm{el}}}{\partial \widetilde{\boldsymbol{D}}_{j}} \widetilde{\mathrm{el}}_{\mathrm{e}-\mathrm{el}}^{-1} \widetilde{\mathbb{D}}_{\mathrm{e} 1-\mathrm{st}} \quad j=1, \ldots, m^{\mathrm{c}}, \quad \text { (B-4) }
$$

where

$$
\frac{\partial \widetilde{\mathbb{D}}_{\mathrm{el}-\mathrm{el}}}{\partial \widetilde{\omega}_{j}}=2 \widetilde{\omega}_{j}(1+\mathrm{i} \eta) \operatorname{diag}\left\{\delta_{j k}\right\}_{k=1, \ldots m^{\mathrm{c}}} \quad j=1, \ldots, m^{\mathrm{c}} .
$$

To derive Eq. (B-5), the modal mass has been assessed implicitly as $\widetilde{\gamma}_{j}=1 \forall j$ (see subsection 3.2). 


\section{References}

[1] R. Ohayon, C. Soize, Structural Acoustics and Vibration, Academic Press, San Diego, 1998.

[2] J.-M. Mencik, M. N. Ichchou, A substructuring technique for finite element wave propagation in multi-layered systems, Computer Methods in Applied Mechanics and Engineering 197 (6-8) (2008) 505-523.

[3] J.-M. Mencik, On the low- and mid-frequency forced response of elastic systems using wave finite elements with one-dimensional propagation, Computers and Structures 88 (11-12) (2010) 674-689.

[4] Y. Waki, B. Mace, M. Brennan, Numerical issues concerning the wave and finite element method for free and forced vibrations of waveguides, Journal of Sound and Vibration 327 (1-2) (2009) 92-108.

[5] Y. Waki, B. Mace, M. Brennan, Free and forced vibrations of a tyre using a wave/finite element approach, Journal of Sound and Vibration 323 (3-5) (2009) 737-756.

[6] W. X. Zhong, F. W. Williams, On the direct solution of wave propagation for repetitive structures, Journal of Sound and Vibration 181 (3) (1995) 485-501.

[7] K. C. Park, C. A. Felippa, R. Ohayon, Reduced-order partitioned modeling of coupled systems: Formulation and computational algorithms, Multi-physics and Multi-scale Computer Models in Non-linear Analysis and Optimal Design of Engineering Structures Under Extreme Conditions (2004) 50-110.

[8] Z. Bai, Krylov subspace techniques for reduced-order modeling of large-scale dynamical systems, Applied Numerical Mathematics 43 (2002) 9-44. 
[9] K. Willcox, J. Peraire, Balanced model reduction via the proper orthogonal decomposition, AIAA Journal 40 (11) (2002) 2323-2330.

[10] J.-M. Mencik, M. N. Ichchou, Multi-mode propagation and diffusion in structures through finite elements, European Journal of Mechanics - A/Solids 24 (5) (2005) 877-898.

[11] W. J. Zhou, M. N. Ichchou, J.-M. Mencik, Analysis of wave propagation in cylindrical pipes with local inhomogeneities, Journal of Sound and Vibration 319 (2009) 335-354.

[12] S. Gopalakrishnan, J. F. Doyle, Spectral super-elements for wave propagation in structures with local non-uniformities, Computer Methods in Applied Mechanics and Engineering 121 (1995) 77-90.

[13] B.-S. Liao, Z. Bai, W. Gao, The important modes of subsystems: A momentmatching approach, International Journal for Numerical Methods in Engineering 70 (13) (2007) 1581-1597.

[14] P. E. Barbone, D. Givoli, I. Patlashenko, Optimal modal reduction of vibrating structures, International Journal for Numerical Methods in Engineering 57 (2003) 341-369.

[15] D. Givoli, P. E. Barbone, I. Patlashenko, Which are the important modes of a subsystem?, International Journal for Numerical Methods in Engineering 59 (2004) 1657-1678.

[16] H. D. Gersem, D. Moens, W. Desmet, D. Vandepitte, Interval and fuzzy dynamic analysis of finite element models with superelements, Computers and Structures 85 (2007) 304-319. 
[17] M. N. Ichchou, J.-M. Mencik, W. J. Zhou, Wave finite elements for low and mid-frequency description of coupled structures with damage, Computer Methods in Applied Mechanics and Engineering 198 (15-16) (2009) 13111326.

[18] D. J. Mead, A general theory of harmonic wave propagation in linear periodic systems with multiple coupling, Journal of Sound and Vibration 27 (2) (1973) 235-260.

[19] C. Wilcox, Theory of Bloch waves, Journal d'Analyse Mathématique 33.

[20] C. Bernardi, Y. Maday, A. T. Patera, A new nonconforming approach to domain decomposition: the mortar element method, Nonlinear Partial Differential Equations and Their Applications, Pitman and Wiley: New York.

[21] R. H. Lyon, R. G. DeJong, Theory and Application of Statistical Energy Analysis, Butterworth-Heinemann, Boston, 1995.

[22] R. S. Langley, P. Bremner, A hybrid method for the vibration analysis of complex structural-acoustic systems, Journal of the Acoustical Society of America 105 (3) (1999) 1657-1671.

[23] Y. C. Fung, Foundations of Solid Mechanics, Prentice Hall, Inc., Englewoods Cliffs, New Jersey, 2003.

[24] G. J. Golub, C. F. V. Loan, Matrix Computations, The Johns Hopkins University Press, Baltimore and London, 1996.

[25] J.-M. Mencik, A. Berry, A diffusion model for a one-dimensional structure coupled with an auxiliary system, Journal of Sound and Vibration 294 (4-5) (2006) 894-915. 
[26] R. R. Craig, M. C. C. Bampton, Coupling of substructures for dynamic analyses, AIAA Journal 6 (7) (1968) 1313-1319.

[27] S. Timoshenko, D. H. Young, W. Weaver, Vibration Problem in Engineering, John Wiley and Sons, Inc, 1990.

[28] G. W. Stewart, On the perturbation of pseudo-inverses, projections and linear least squares problems, SIAM Review 19 (4) (1977) 634-662.

[29] P. A. Wedin, Perturbation theory for pseudo-inverses, BIT Numerical Mathematics 13 (2) (1973) 217-232.

[30] N. J. Higham, A survey of componentwise perturbation theory, Proceedings of Symposia in Applied Mathematics, American Mathematical Society, Providence, RI, USA (1994) 49-77.

[31] S. Gugercin, An iterative SVD-Krylov based method for model reduction of large-scale dynamical systems, Linear Algebra and its Applications 428 (2008) 1964-1986.

[32] R. S. Langley, Wave evolution, reflection, and transmission along inhomogeneous waveguides, Journal of Sound and Vibration 227 (1) (1999) 131-158. 


\section{List of Figures}

1 Neumann-to-Dirichlet problem involving two waveguides with an elastic coupling junction. . . . . . . . . . . . . . . . . 46

2 Illustration of incident / reflected waves; finite element models of two connected substructures $k-1$ and $k \ldots \ldots \ldots$. . . . . 47

3 Spatial representation of several "cross-section" wave shapes at $2500 \mathrm{~Hz}$ (the direction of propagation is indicated by an arrow): (a) flexural mode; (b) torsional mode; (c) longitudinal mode; (d) shearing mode; (e-i) MF modes. . . . . . . . . . . . . . . . . . 48

4 Mesh tying problem considered in the WFE framework: junction with two connected substructures. . . . . . . . . . . . . . . . 49

5 Frequency response of the coupled system depicted in Figure 1: (-) solution provided by FE; (-॰-) solutions provided by WFE with $m=10$ wave modes (a), $m=20$ wave modes (b), $m=40$ wave modes (c), $m=50$ wave modes $(\mathrm{d}) \ldots \ldots \ldots$

6 Frequency evolution of reflection (-) and transmission (- - -) coefficients (real parts) among flexural, torsional and MF modes. . . 51

7 Frequency evolution of power reflection (-) and transmission (- -) coefficients for incident flexural mode. . . . . . . . . . . . 52

8 Frequency evolution of power reflection (-) and transmission (- -) coefficients for incident torsional mode. . . . . . . . . . . 53

9 Eigenfrequencies of first 30 junction modes; (-) upper frequency limit (i.e. $5000 \mathrm{~Hz}$ ) of $\mathcal{B}_{f}$. . . . . . . . . . . . . . . . . . 54 
10 Frequency response of the coupled system depicted in Figure 1: (-) solution provided by FE; (-๑-) solutions provided by WFE with 50 wave modes and using CMS with $m^{\mathrm{c}}=0$ junction modes (a), $m^{\mathrm{c}}=10$ junction modes (b), $m^{\mathrm{c}}=20$ junction modes (c) and $m^{\mathrm{c}}=30$ junction modes $(\mathrm{d}) \ldots \ldots \ldots \ldots \ldots$

11 Bounds provided by Eq. (37) for the first 30 junction modes; (-) threshold of $10 \%$ above which the junction modes are selected. . . 56

12 Frequency response of the coupled system depicted in Figure 1: (-) solution provided by FE; (-॰-) solutions provided by CMSbased WFE with $m^{\mathrm{c}}=19$ junction modes selected by means of

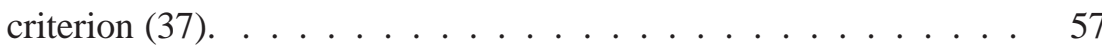

13 Relative error $\mathcal{E}^{\mathrm{WFE}}$ with ascending order strategy (-) and criterion (37) (- - ) (the violet line indicates that 19 junction modes are selected).

14 Frequency response of the coupled system depicted in Figure 1: $(-\bullet-)$ baseline solution provided by CMS-based WFE with $m^{\mathrm{c}}=$ 19 junction modes (a); (-) MCS solutions with 100 trials (b); (yellow shaded area: (a) and (b)) perturbation bounds obtained by means of Eq. (48). . . . . . . . . . . . . . . . . . . . 59 


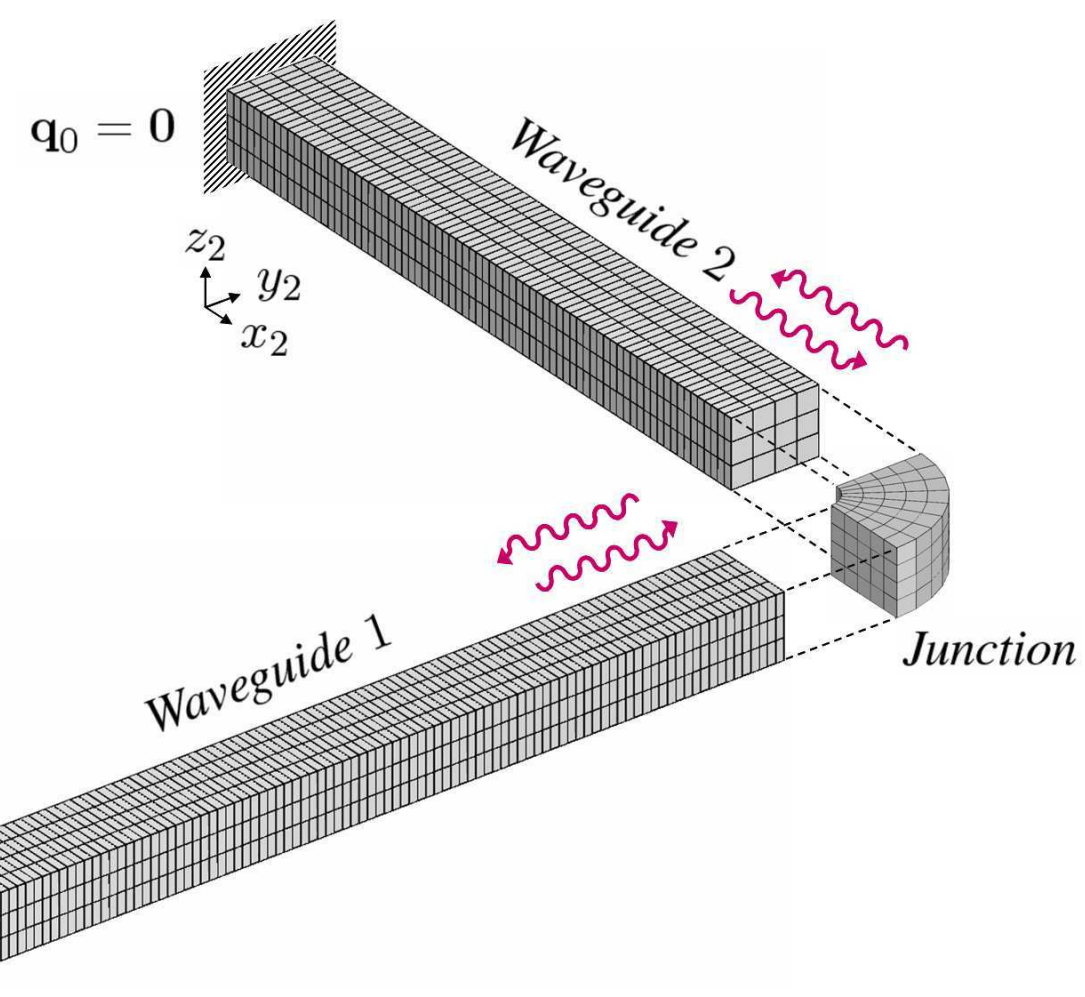

Figure 1: Neumann-to-Dirichlet problem involving two waveguides with an elastic coupling junction. 


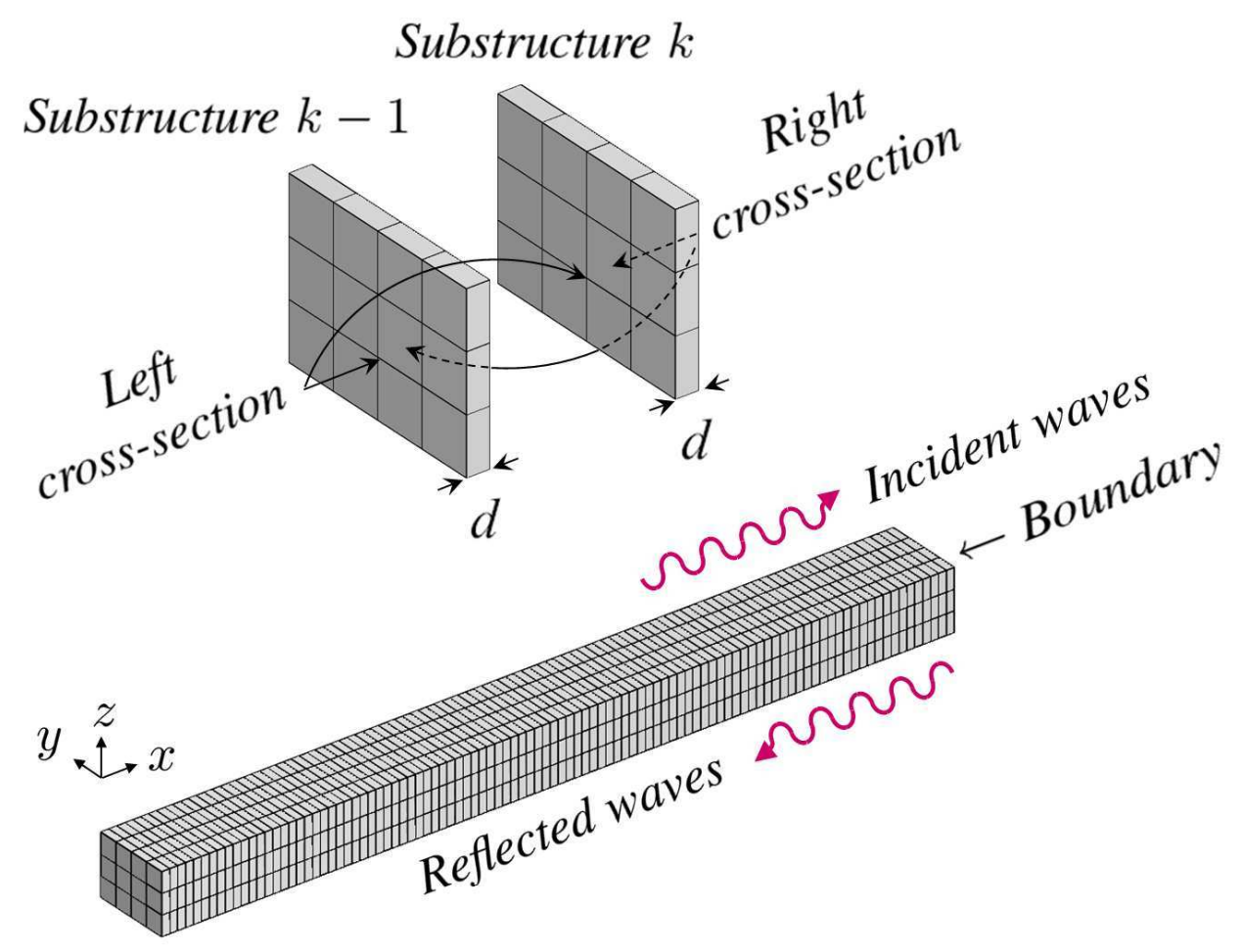

Figure 2: Illustration of incident / reflected waves; finite element models of two connected substructures $k-1$ and $k$. 
(a)

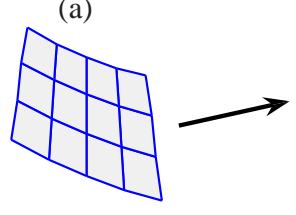

(d)

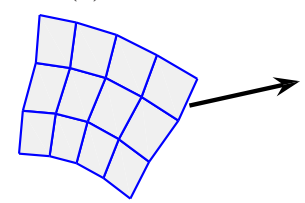

(g)

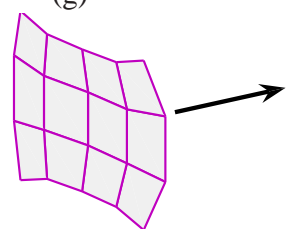

(b)

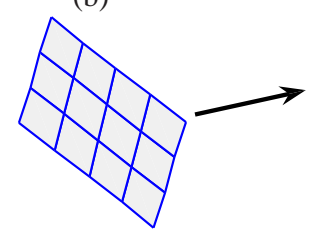

(e)

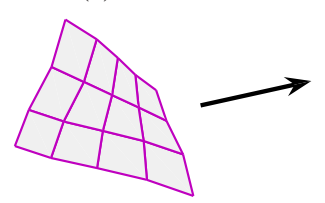

(h)

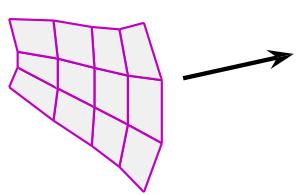

(c)

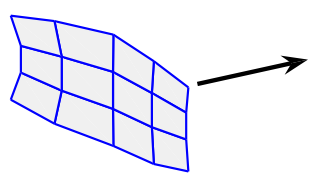

(f)

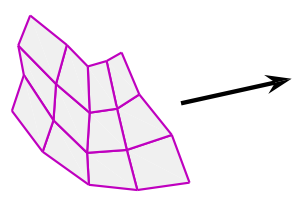

(i)

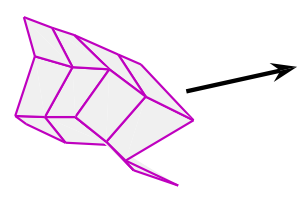

Figure 3: Spatial representation of several "cross-section" wave shapes at $2500 \mathrm{~Hz}$ (the direction of propagation is indicated by an arrow): (a) flexural mode; (b) torsional mode; (c) longitudinal mode; (d) shearing mode; (e-i) MF modes. 


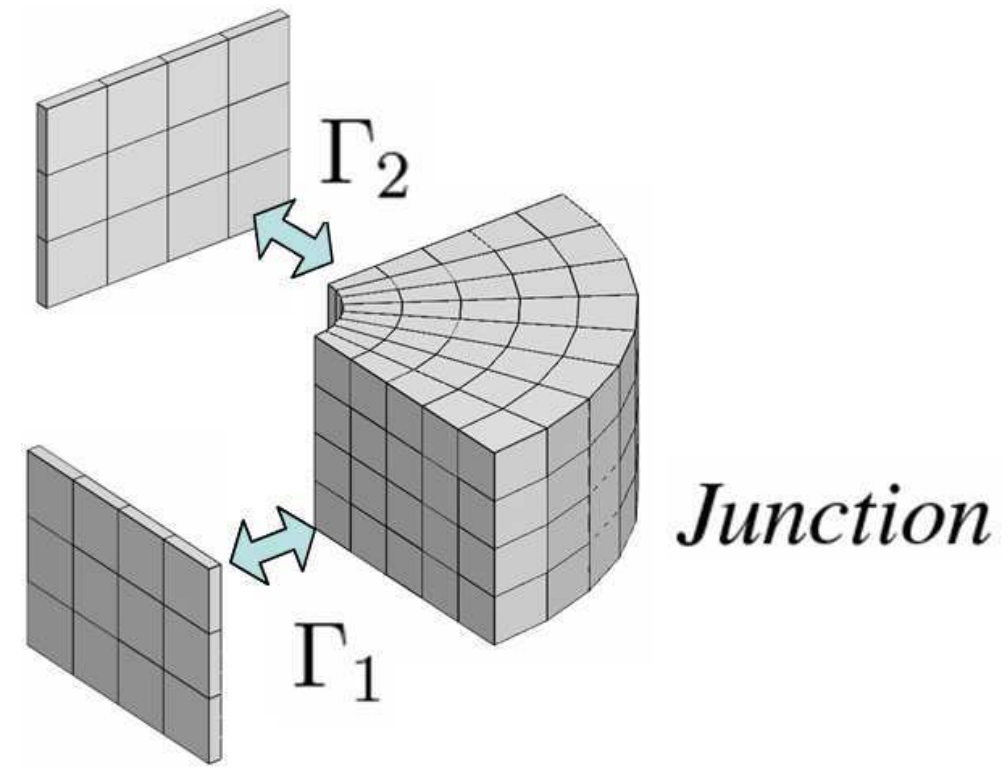

Figure 4: Mesh tying problem considered in the WFE framework: junction with two connected substructures. 

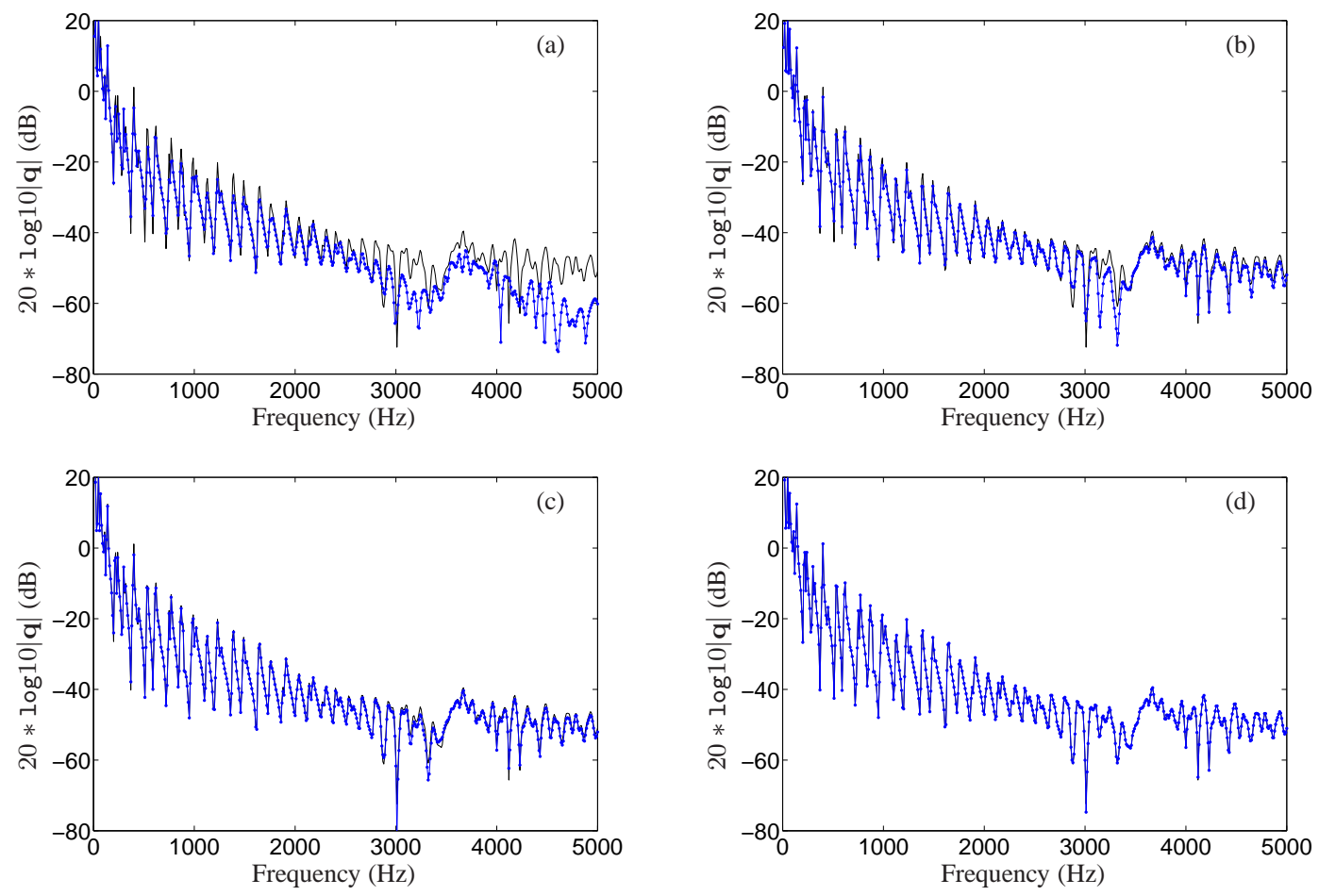

Figure 5: Frequency response of the coupled system depicted in Figure 1: (-) solution provided by FE; (-๑-) solutions provided by WFE with $m=10$ wave modes (a), $m=20$ wave modes (b), $m=40$ wave modes (c), $m=50$ wave modes (d). 

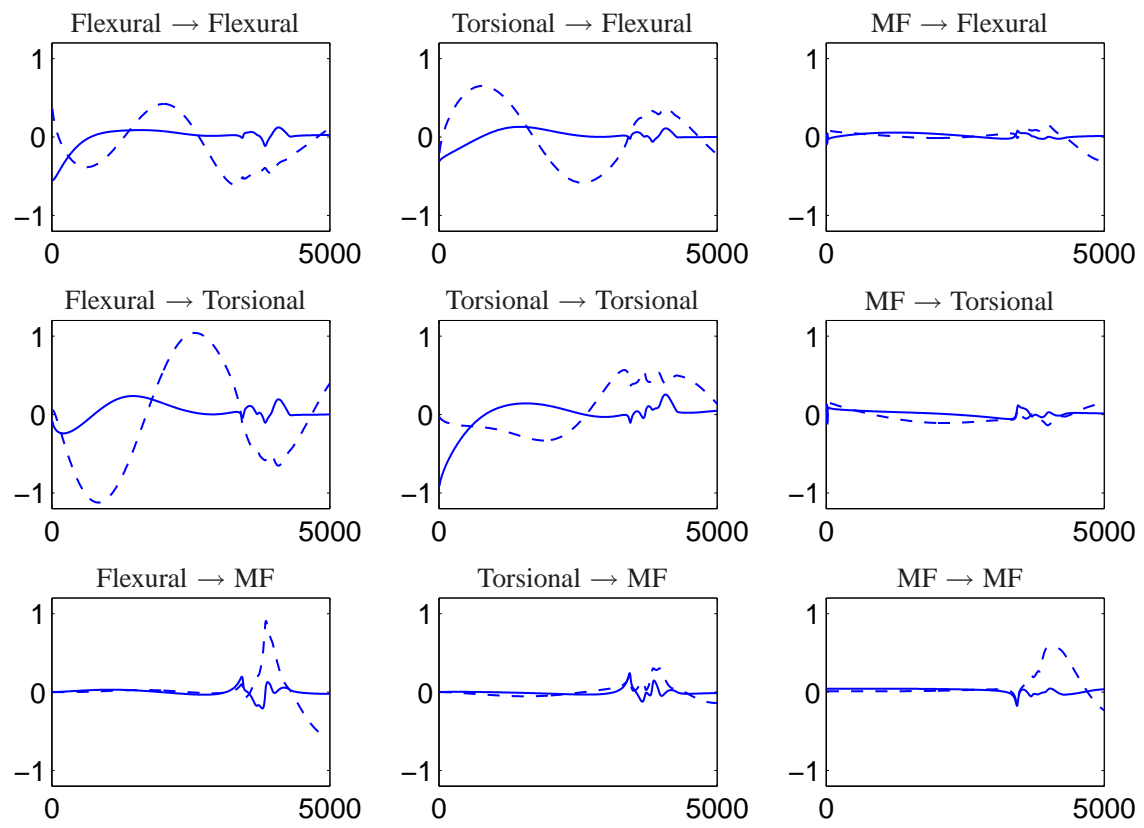

Figure 6: Frequency evolution of reflection (-) and transmission (- - -) coefficients (real parts) among flexural, torsional and MF modes. 

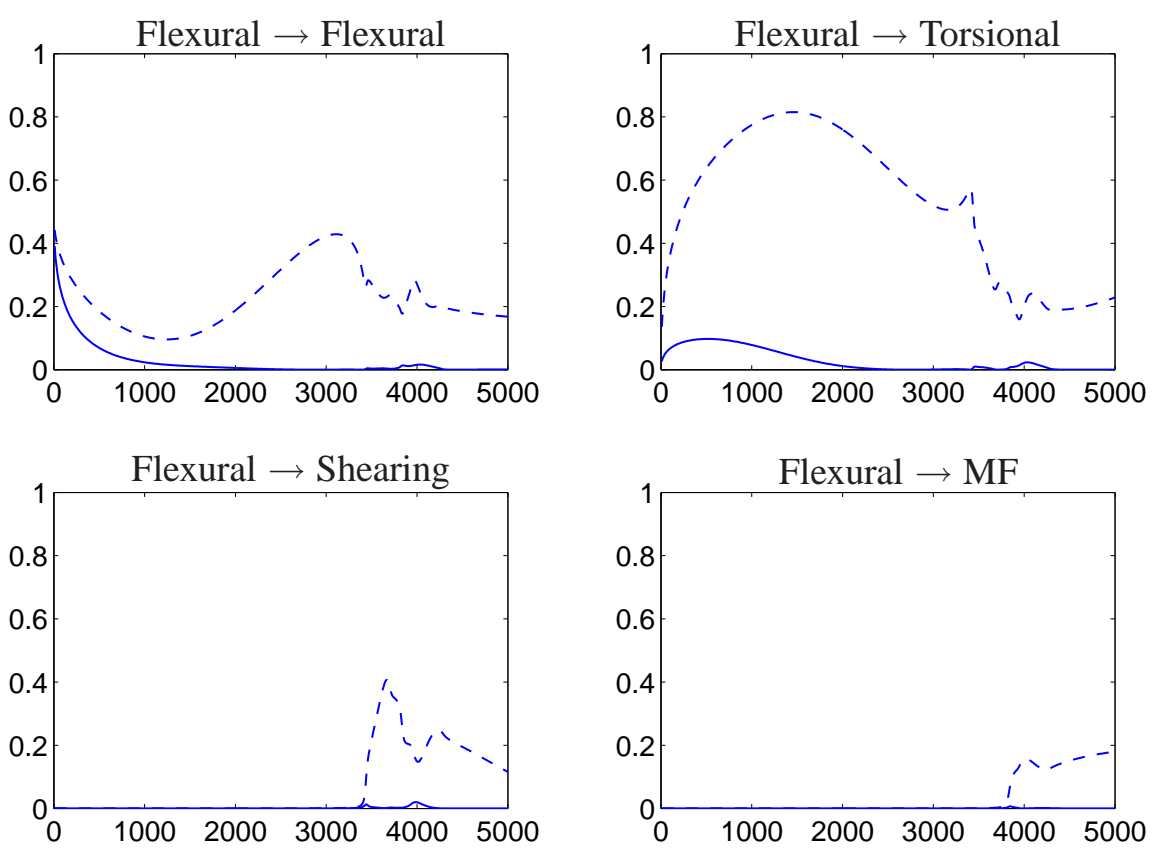

Figure 7: Frequency evolution of power reflection (-) and transmission (- - ) coefficients for incident flexural mode. 

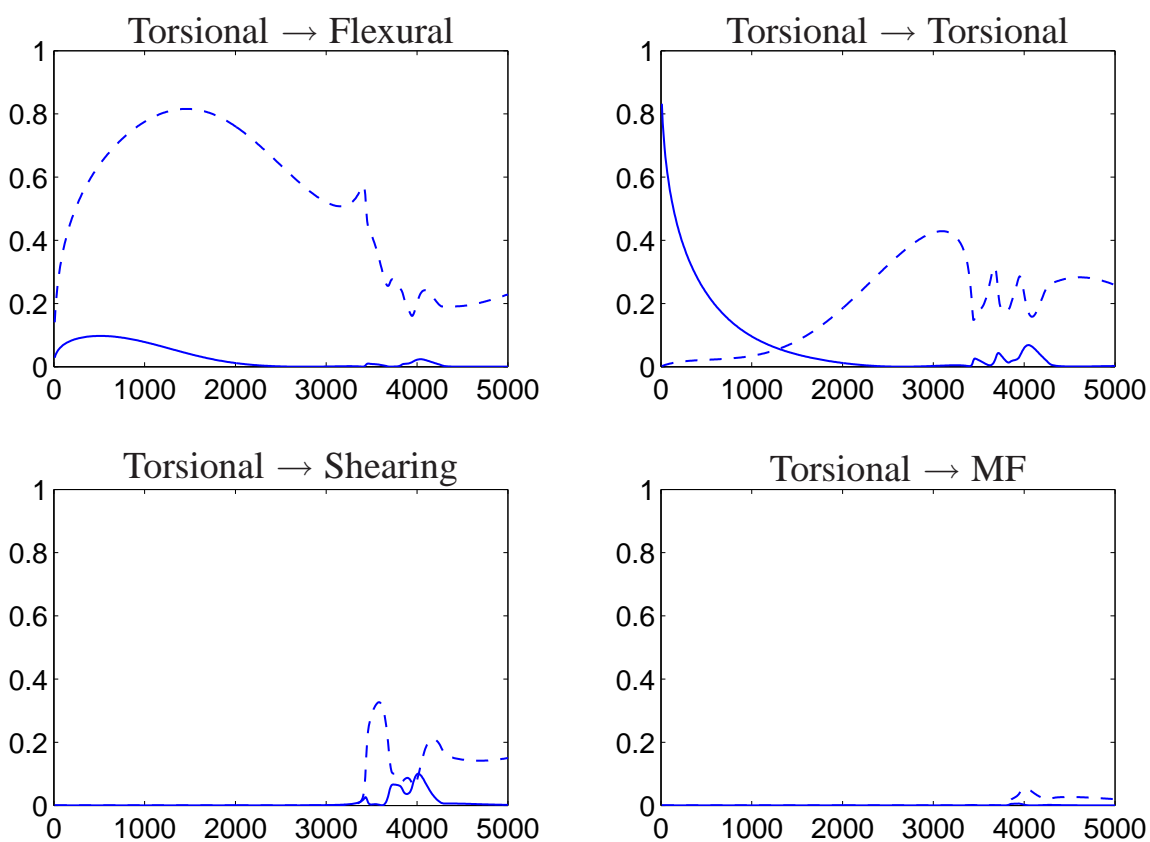

Figure 8: Frequency evolution of power reflection (-) and transmission (- - -) coefficients for incident torsional mode. 


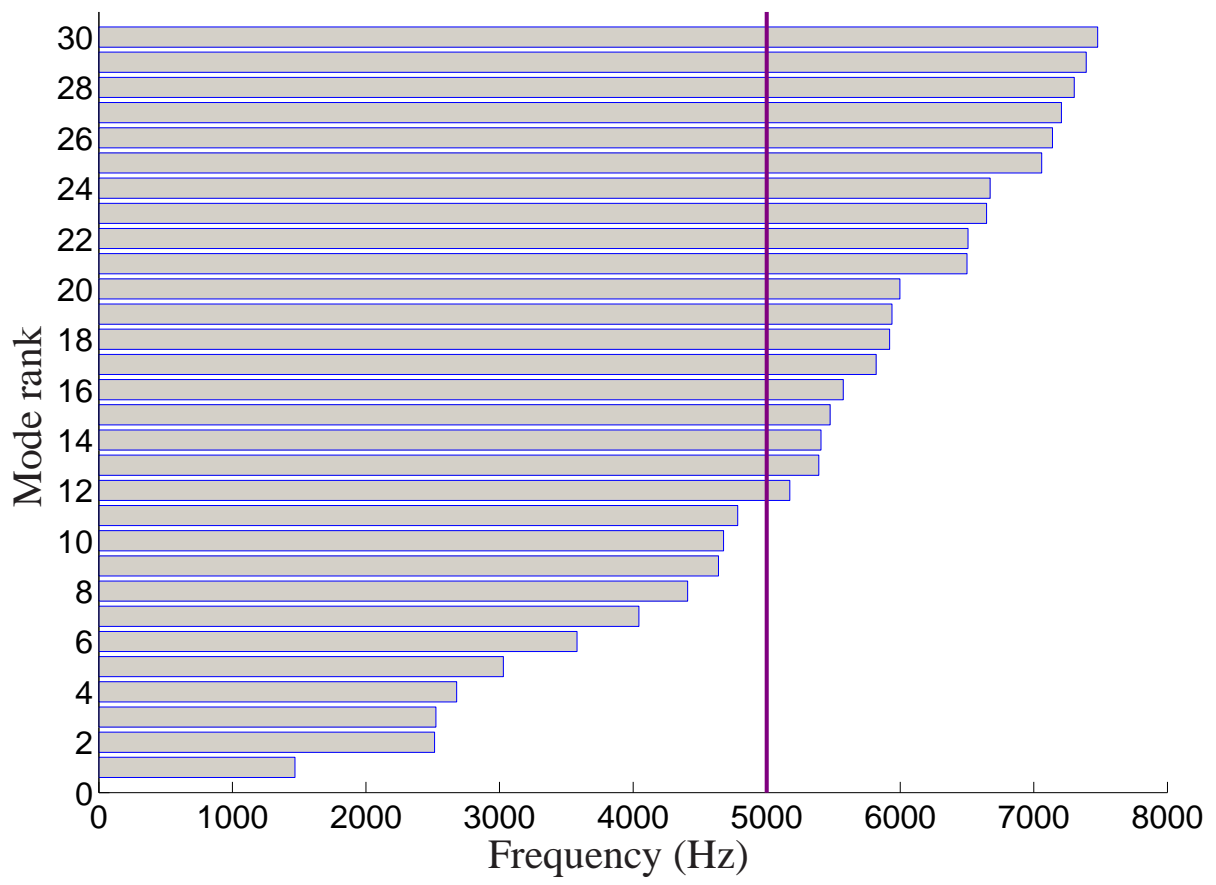

Figure 9: Eigenfrequencies of first 30 junction modes; (-) upper frequency limit (i.e. $5000 \mathrm{~Hz}$ ) of $\mathcal{B}_{f}$. 

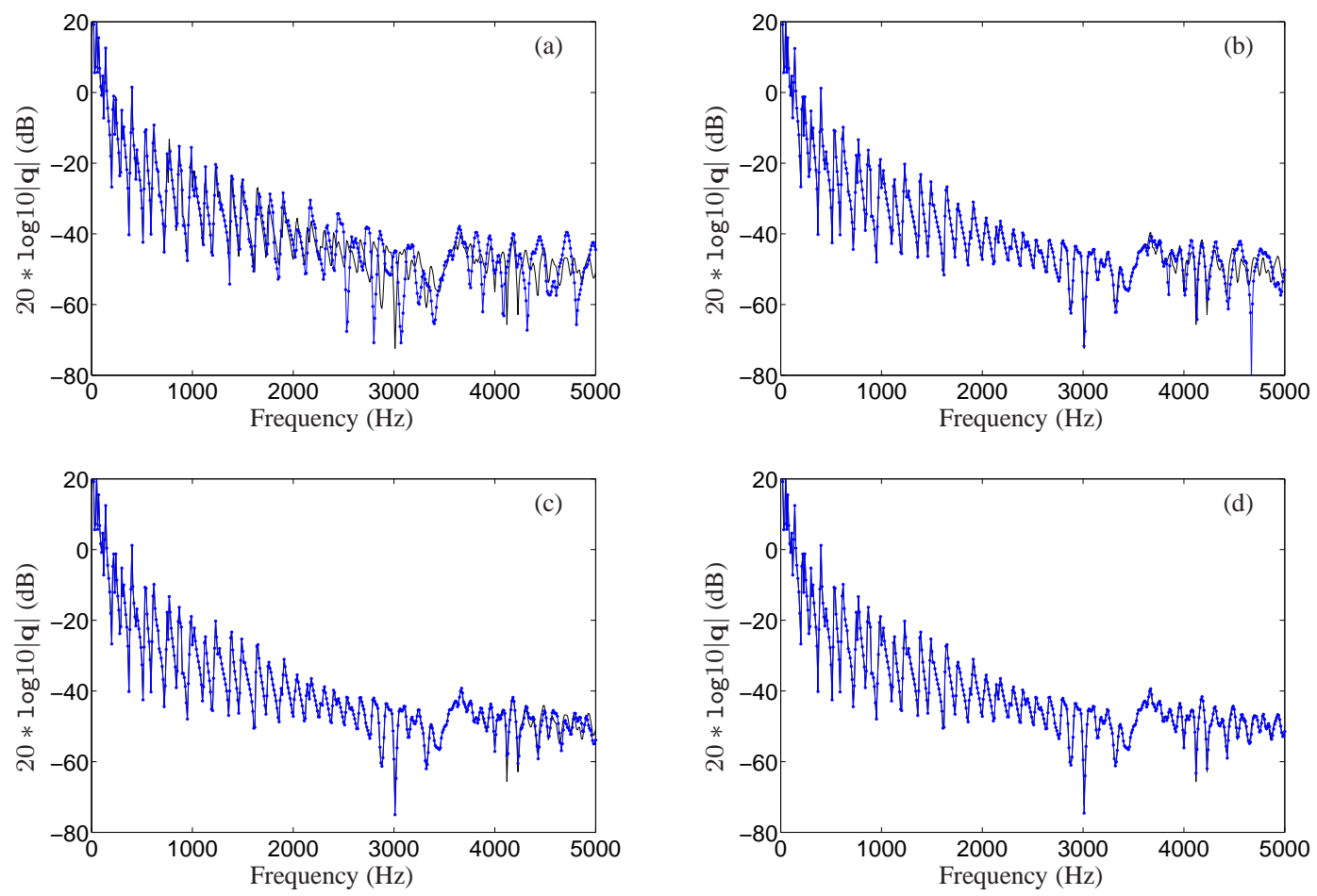

Figure 10: Frequency response of the coupled system depicted in Figure 1: (-) solution provided by FE; (-๑-) solutions provided by WFE with 50 wave modes and using CMS with $m^{\mathrm{c}}=0$ junction modes (a), $m^{c}=10$ junction modes (b), $m^{c}=20$ junction modes (c) and $m^{c}=30$ junction modes (d). 


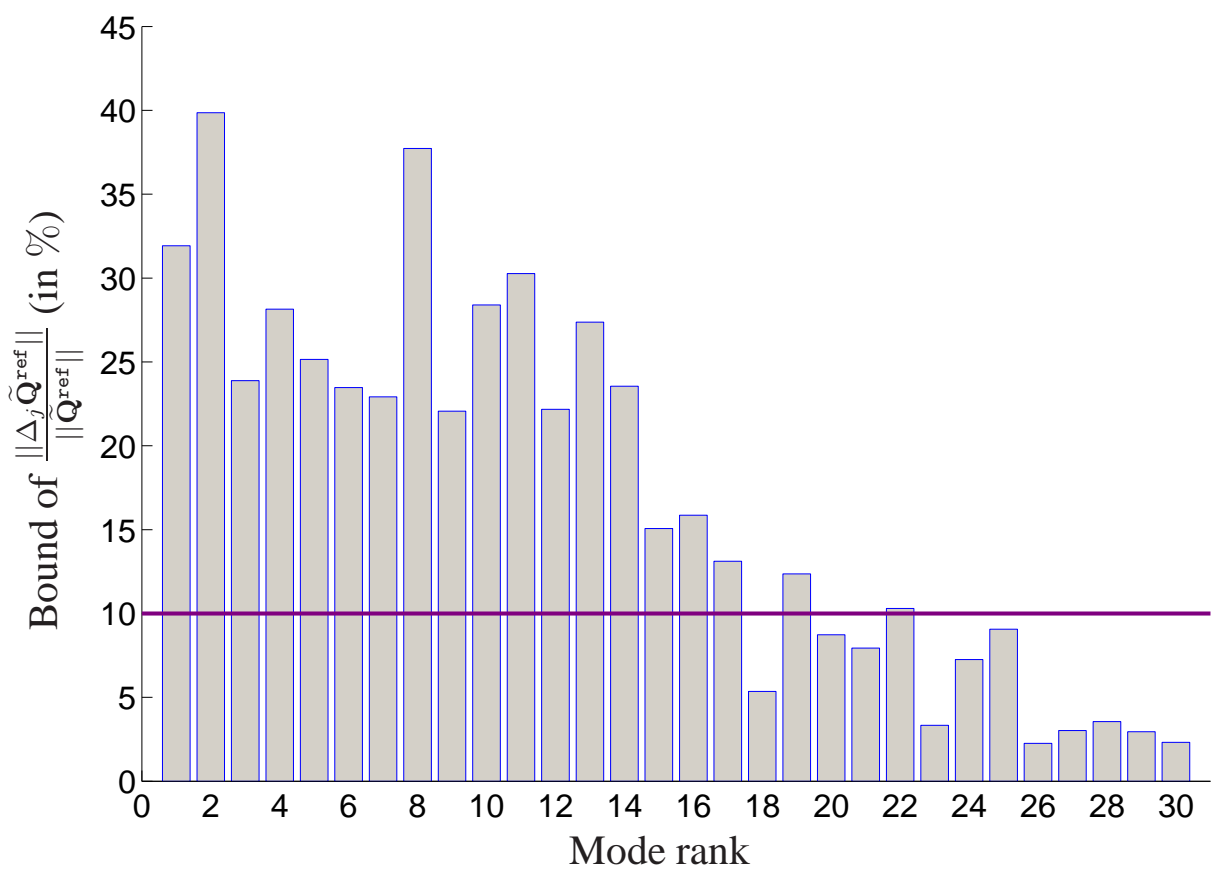

Figure 11: Bounds provided by Eq. (37) for the first 30 junction modes; (-) threshold of $10 \%$ above which the junction modes are selected. 


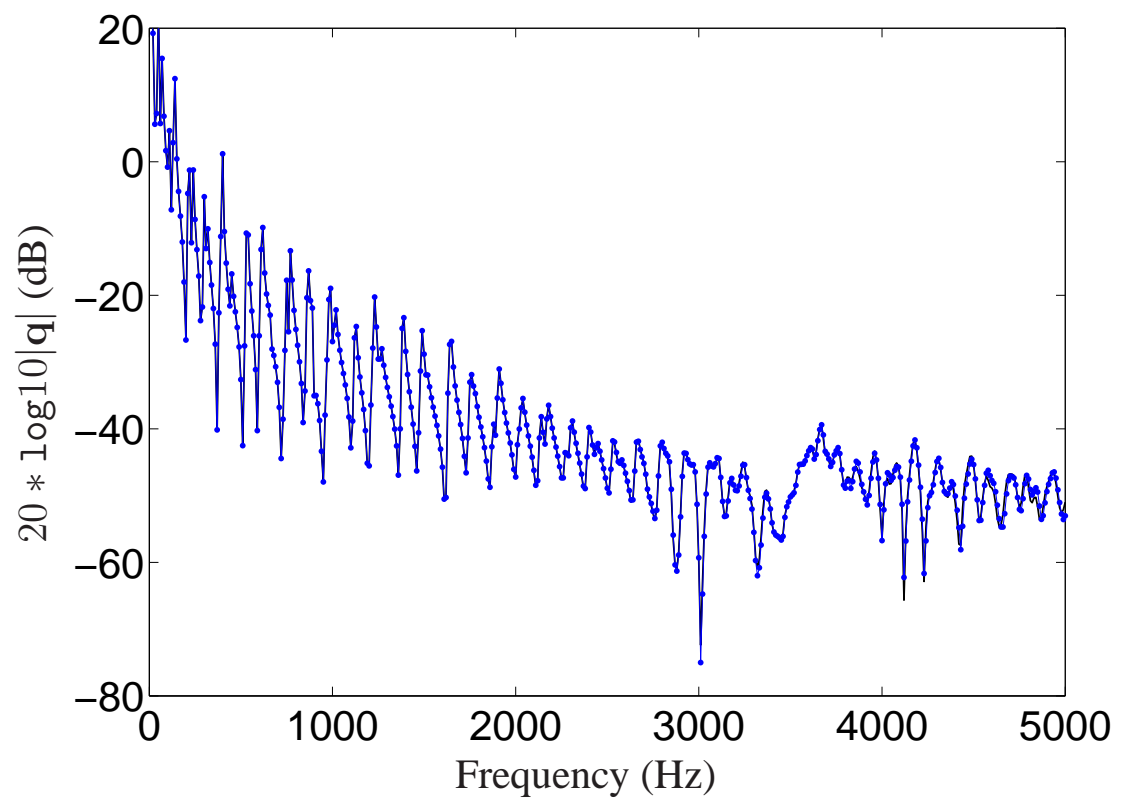

Figure 12: Frequency response of the coupled system depicted in Figure 1: (-) solution provided by FE; (-๑-) solutions provided by CMS-based WFE with $m^{c}=19$ junction modes selected by means of criterion (37). 


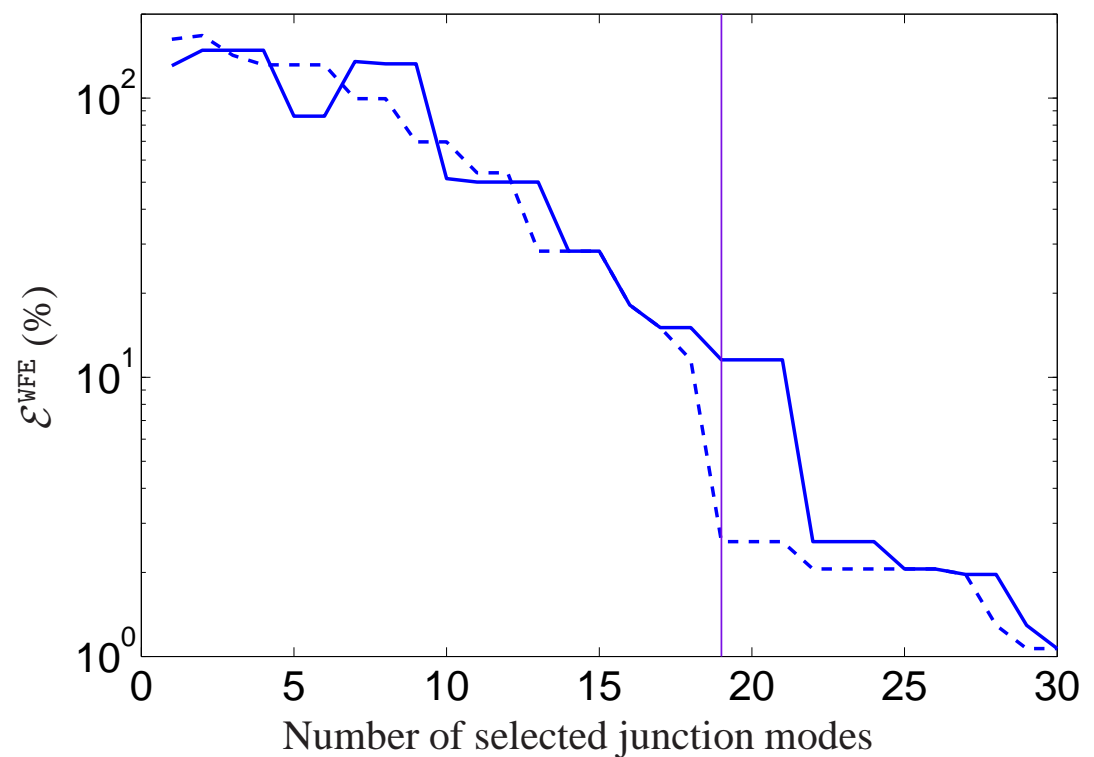

Figure 13: Relative error $\mathcal{E}^{\mathrm{WFE}}$ with ascending order strategy (- ) and criterion (37) (- - -) (the violet line indicates that 19 junction modes are selected). 

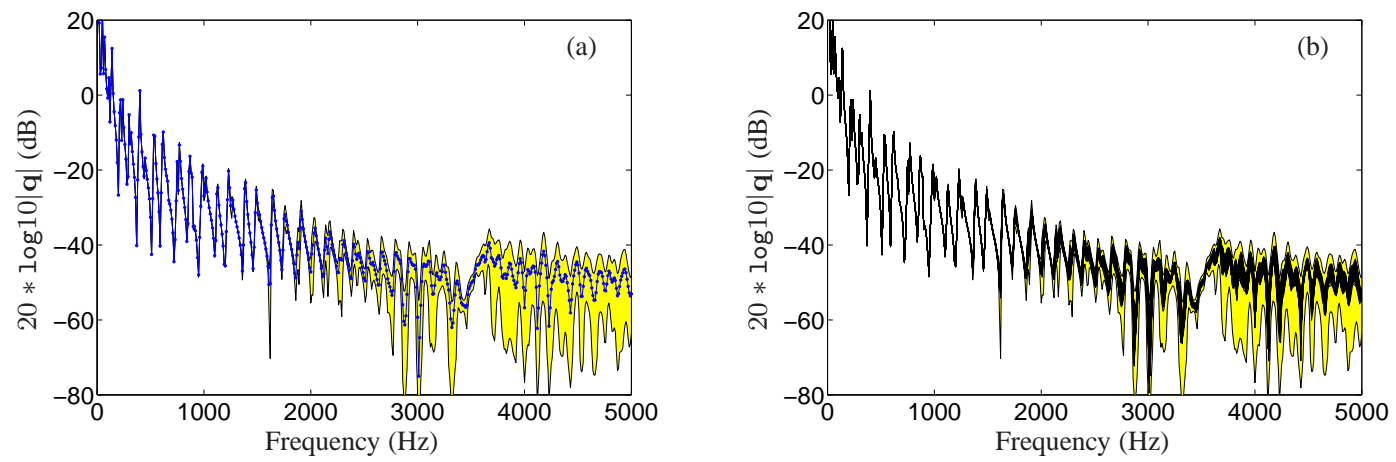

Figure 14: Frequency response of the coupled system depicted in Figure 1: (-๑-) baseline solution provided by CMS-based WFE with $m^{c}=19$ junction modes (a); (-) MCS solutions with 100 trials (b); (yellow shaded area: (a) and (b)) perturbation bounds obtained by means of Eq. (48). 